\title{
Resummation of Feynman Diagrams and the Inversion of Matrices
}

by

\author{
Detlef Lehmann円 \\ Technische Universität Berlin \\ Fachbereich Mathematik Ma 7-2 \\ Staße des 17. Juni 136 \\ D-10623 Berlin, GERMANY
}

\begin{abstract}
In many field theoretical models one has to resum two- and four-legged subdiagrams in order to determine their behaviour. In this article we present a novel formalism which does this in a nice way. It is based on the central limit theorem of probability and an inversion formula for matrices which is obtained by repeated application of the Feshbach projection method. We discuss applications to the Anderson model, to the many-electron system and to the $\varphi^{4}$-model. In particular, for the many-electron system with attractive delta-interaction, we find that the existence of a BCS gap and a macroscopic value of the Hubbard-Stratonovich field for zero momentum enforce each other.
\end{abstract}

\footnotetext{
1 e-mail: lehmann@math.tu-berlin.de
} 


\section{Introduction}

The computation of correlation functions in field theoretical models is a difficult problem. In this article we present a novel approach which applies to models where a two point function can be written as

$$
S(x, y)=\int[P+Q]_{x, y}^{-1} d \mu(Q)
$$

Here $P$ is some operator diagonal in momentum space, typically determined by the unperturbed Hamiltonian, and $Q$ is diagonal in coordinate space. The functional integral is taken with respect to some probability measure $d \mu(Q)$ and goes over the matrix elements of $Q \cdot[\cdot]_{x, y}^{-1}$ denotes the $x, y$-entry of the matrix $[P+Q]^{-1}$. Our starting point is always a model in finite volume and with positive lattice spacing in which case the operator $P+Q$ and the functional integral in (1.1) becomes huge- but finite-dimensional. In the end we take the infinite volume limit and, if wanted, the continuum limit.

Our treatment is based on the following identity which is obtained by repeated application of the Feshbach formula (Lemma 3.2 below). It is proven in Theorem 3.3. Let $B=\left(B_{k p}\right)_{k, p \in \mathcal{M}} \in \mathbb{C}^{N \times N}, \mathcal{M}$ some index set, $|\mathcal{M}|=N$ and let

$$
G(k):=\left[B^{-1}\right]_{k k}
$$

Then one has

$$
G(k)=\frac{1}{B_{k k}+\sum_{r=2}^{N}(-1)^{r+1} \sum_{\substack{p_{2} \cdots p_{r} \in \mathcal{M} \backslash\{k\} \\ p_{i} \neq p_{j}}} B_{k p_{2}} G_{k}\left(p_{2}\right) B_{p_{2} p_{3}} \cdots B_{p_{r-1} p_{r}} G_{k p_{2} \cdots p_{r-1}}\left(p_{r}\right) B_{p_{r} k}}
$$

where $G_{k_{1} \cdots k_{j}}(p)=\left[\left(B_{s t}\right)_{s, t \in \mathcal{M} \backslash\left\{k_{1} \cdots k_{j}\right\}}\right]_{p p}^{-1}$ is the $p, p$ entry of the inverse of the matrix which is obtained from $B$ by deleting the rows and columns labelled by $k_{1}, \cdots, k_{j}$. In Section 2 we apply this formula to a matrix of the form $B=$ self adjoint $+i \varepsilon I d$, which, for $\varepsilon \neq 0$, has the property that all submatrices $\left(B_{s t}\right)_{s, t \in \mathcal{M} \backslash\left\{k_{1} \cdots k_{j}\right\}}$ are invertible.

There is also a formula for the off-diagonal inverse matrix elements. It reads

$$
\left[B^{-1}\right]_{k p}=-G(k) B_{k p} G_{k}(p)+\sum_{r=3}^{N}(-1)^{r+1} \sum_{\substack{t_{3} \cdots t_{r} \in \mathcal{M} \backslash\{k, p\} \\ t_{i} \neq t_{j}}} G(k) B_{k t_{3}} G_{k}\left(t_{3}\right) B_{t_{3} t_{4}} \cdots B_{t_{r} p} G_{k t_{3} \cdots t_{r}}(p)
$$

These formulae also hold in the case where the matrix $B$ has a block structure $B_{k p}=$ $\left(B_{k \sigma, p \tau}\right)$ where, say, $\sigma, \tau \in\{\uparrow, \downarrow\}$ are some spin variables. In that case the $B_{k p}$ are small matrices, the $G_{k_{1} \cdots k_{j}}(p)$ are matrices of the same size and the $1 / \cdot$ in $(1.3)$ means inversion of matrices, see Theorem 3.3 below. 
The paper is organized as follows. In the next section we demonstrate the method by applying it to the averaged Green function of the Anderson model. The Schwinger-Dyson equation for that model reads $G^{-1}=G_{0}^{-1}+\Sigma\left(G_{0}\right)$ where $\Sigma\left(G_{0}\right)$ is the sum of all two-legged one-particle irreducible diagrams. Application of (1.3) leads to an integral equation $G^{-1}=$ $G_{0}^{-1}+\sigma(G)$ where $\sigma(G)$ is the sum of all two-legged graphs without two-legged subgraphs. The latter equation has two advantages. First, $\Sigma$ is the sum of one-particle irreducible diagrams, but these diagrams may very well have two-legged subdiagrams and usually these are the diagrams which produce anomalously large contributions. And second, the propagator for $\sigma(G)$ is the interacting two point function $G$, which, for the Anderson model, is more regular than the free two point function $G_{0}$ which is the propagator for the diagrams contributing to $\Sigma\left(G_{0}\right)$. More precisely, the series for $\sigma(G)$ can be expected to be asymptotic, that is, its lowest order contributions are a good approximation if the coupling is small, but, usually, the series for $\Sigma\left(G_{0}\right)$ is not asymptotic.

For the many-electron system and for the $\varphi^{4}$ model repeated application of $(1.3,4)$ amounts to a resummation of two- and four-legged subgraphs. This is discussed in section 4. In section 5 we discuss how our method is related to the integral equations which can be found in the literature. The proof of the inversion formula is given in section 3 .

\section{Application to the Anderson Model}

Let coordinate space be a lattice of finite volume with periodic boundary conditions, lattice spacing $1 / M$ and volume $[0, L]^{d}$ :

$$
\Gamma=\left\{x=\frac{1}{M}\left(n_{1}, \cdots, n_{d}\right) \mid 0 \leq n_{i} \leq M L-1\right\}=\left(\frac{1}{M} \mathbb{Z}\right)^{d} /(L \mathbb{Z})^{d}
$$

Momentum space is given by

$$
\mathcal{M}:=\Gamma^{\sharp}=\left\{k=\frac{2 \pi}{L}\left(m_{1}, \cdots, m_{d}\right) \mid 0 \leq m_{i} \leq M L-1\right\}=\left(\frac{2 \pi}{L} \mathbb{Z}\right)^{d} /(2 \pi M \mathbb{Z})^{d}
$$

We consider the averaged Green function of the Anderson model given by

$$
\langle G\rangle\left(x, x^{\prime}\right):=\int[-\Delta-z+\lambda V]_{x, x^{\prime}}^{-1} d P(V)
$$

where the random potential is Gaussian,

$$
d P(V)=\prod_{x \in \Gamma} e^{-\frac{V_{x}^{2}}{2}} \frac{d V_{x}}{\sqrt{2 \pi}} .
$$

Here $z=E+i \varepsilon$ and $\Delta$ is the discrete Laplacian,

$$
[-\Delta-z+\lambda V]_{x, x^{\prime}}=-M^{2} \sum_{i=1}^{d}\left(\delta_{x^{\prime}, x+e_{i} / M}+\delta_{x^{\prime}, x-e_{i} / M}-2 \delta_{x^{\prime}, x}\right)-z \delta_{x, x^{\prime}}+\lambda V_{x} \delta_{x, x^{\prime}}
$$


By taking the Fourier transform, one has

$$
\begin{aligned}
\langle G\rangle\left(x, x^{\prime}\right) & =\frac{1}{M^{d} L^{d}} \sum_{k \in \mathcal{M}} e^{i k\left(x^{\prime}-x\right)}\langle G\rangle(k) \\
\langle G\rangle(k) & =\int_{\mathbb{R}^{N^{d}}}\left[a_{k} \delta_{k, p}+\frac{\lambda}{\sqrt{N^{d}}} v_{k-p}\right]_{k, k}^{-1} d P(v)
\end{aligned}
$$

where $N^{d}=(M L)^{d}=|\Gamma|=|\mathcal{M}|, d P(v)$ is given by (2.10) or (2.11) below, depending on whether $N^{d}$ is even or odd, and

$$
a_{k}=4 M^{2} \sum_{i=1}^{d} \sin ^{2}\left[\frac{k_{i}}{2 M}\right]-E-i \varepsilon
$$

The rigorous control of $\langle G\rangle(k)$ for small disorder $\lambda$ and energies inside the spectrum of the unperturbed Laplacian, $E \in\left[0,4 M^{2}\right]$, in which case $a_{k}$ has a root if $\varepsilon \rightarrow 0$, is still an open problem $[\mathrm{AG}, \mathrm{K}, \mathrm{MPR}, \mathrm{P}, \mathrm{W}]$. It is expected that $\lim _{\varepsilon \searrow 0} \lim _{L \rightarrow \infty}\langle G\rangle(k)=1 /\left(a_{k}-\sigma_{k}\right)$ where $\operatorname{Im} \sigma=O\left(\lambda^{2}\right)$.

The integration variables $v_{q}$ in $(2.7)$ are given by the discrete Fourier transform of the $V_{x}$. In particular, observe that, if $F$ denotes the unitary matrix of discrete Fourier transform, the variables

$$
v_{q} \equiv(F V)_{q}=\frac{1}{\sqrt{N^{d}}} \sum_{x \in \Gamma} e^{-i q x} V_{x}=\left(\frac{M}{L}\right)^{\frac{d}{2}} \frac{1}{M^{d}} \sum_{x \in \Gamma} e^{-i q x} V_{x} \equiv\left(\frac{M}{L}\right)^{\frac{d}{2}} \hat{V}_{q}
$$

would not have a limit if $V_{x}$ would be deterministic and cutoffs are removed, since the $\hat{V}_{q}$ are the quantities which have a limit in that case. But since the $V_{x}$ are integration variables, we choose a unitary transform to keep the integration measure invariant. Observe also that $v_{q}$ is complex, $v_{q}=u_{q}+i w_{q}$. Since $V_{x}$ is real, $u_{-q}=u_{q}$ and $w_{-q}=-w_{q}$. In order to transform $d P(V)$ to momentum space, we have to choose a set $\mathcal{M}^{+} \subset \mathcal{M}$ such that either $q \in \mathcal{M}^{+}$or $-q \in \mathcal{M}^{+}$. If $N$ is odd, the only momentum with $q=-q$ or $w_{q}=0$ is $q=0$. In that case $d P(V)$ becomes

$$
d P(v)=e^{-\frac{u_{0}^{2}}{2}} \frac{d u_{0}}{\sqrt{2 \pi}} \prod_{q \in \mathcal{M}^{+}} e^{-\left(u_{q}^{2}+w_{q}^{2}\right)} \frac{d u_{q} d w_{q}}{\pi}
$$

For even $N$ we get

$$
d P(v)=e^{-\frac{1}{2}\left(u_{0}^{2}+u_{q_{0}}^{2}\right) \frac{d u_{0} d u_{q_{0}}}{2 \pi}} \prod_{q \in \mathcal{M}^{+}} e^{-\left(u_{q}^{2}+w_{q}^{2}\right)} \frac{d u_{q} d w_{q}}{\pi}
$$

where $q_{0}=\frac{2 \pi m}{L}$ is the unique nonzero momentum for which $\frac{2 \pi}{L} m=2 \pi M(1, \cdots, 1)-\frac{2 \pi}{L} m$.

Now we apply the inversion formula (1.3) to the inverse matrix element in

$$
\langle G\rangle(k)=\int_{\mathbb{R}^{N^{d}}}\left[a_{k} \delta_{k, p}+\frac{\lambda}{\sqrt{N^{d}}} v_{k-p}\right]_{k, k}^{-1} d P(v)
$$


We start with the 'two loop approximation', which we define by retaining only the $r=2$ term in the denominator of the right hand side of (1.3),

$$
G(k) \approx \frac{1}{B_{k k}-\sum_{p \in \mathcal{M} \backslash\{k\}} B_{k p} G_{k}(p) B_{p k}}
$$

Thus, let

$$
G(k):=\left[a_{k} \delta_{k, p}+\frac{\lambda}{\sqrt{N^{d}}} v_{k-p}\right]_{k, k}^{-1}=G(k ; v, z)
$$

In the infinite volume limit the spacing $2 \pi / L$ of the momenta becomes arbitrary small. Hence, in computing an inverse matrix element, it should not matter whether a single column and row labelled by some momentum $t$ is absent or not. In other words, in the infinite volume limit one should have

$$
G_{t}(p)=G(p) \text { for } L \rightarrow \infty
$$

and similarly $G_{t_{1} \cdots t_{j}}(p)=G(p)$ as long as $j$ is independent of the volume. We remark however that if the matrix has a block structure, say $B=\left(B_{k \sigma, p \tau}\right)$ with $\sigma, \tau \in\{\uparrow, \downarrow\}$ some spin variables, this structure has to be respected. That is, for a given momentum $k$ all rows and columns labelled by $k \uparrow, k \downarrow$ have to be eliminated, since otherwise (2.14) may not be true.

Thus the two loop approximation gives

$$
G(k)=\frac{1}{a_{k}+\frac{\lambda}{\sqrt{N^{d}}} v_{0}-\frac{\lambda^{2}}{N^{d}} \sum_{p \neq k} v_{k-p} G(p) v_{p-k}}
$$

For large $L$, we can disregard the $\frac{\lambda}{\sqrt{N^{d}}} v_{0}$ term. Introducing $\sigma_{k}=\sigma_{k}(v, z)$ according to

$$
G(k)=: \frac{1}{a_{k}-\sigma_{k}},
$$

we get

$$
\sigma_{k}=\frac{\lambda^{2}}{N^{d}} \sum_{p \neq k} \frac{\left|v_{k-p}\right|^{2}}{a_{p}-\sigma_{p}} \approx \frac{\lambda^{2}}{N^{d}} \sum_{p} \frac{\left|v_{k-p}\right|^{2}}{a_{p}-\sigma_{p}}
$$

and arrive at

$$
\langle G\rangle(k)=\int \frac{1}{a_{k}-\frac{\lambda^{2}}{N^{d}} \sum_{p} \frac{\left|v_{k-p}\right|^{2}}{a_{p}-\frac{\lambda^{2}}{N^{d}} \sum_{t} \frac{\left|v_{p-t}\right|^{2}}{a_{t}-\frac{\lambda^{2}}{N^{d}} \Sigma \cdots}}} d P(v)
$$

Now consider the infinite volume limit $L \rightarrow \infty$ or $N=M L \rightarrow \infty$. By the central limit theorem of probability $\frac{1}{\sqrt{N^{d}}} \sum_{q}\left(\left|v_{q}\right|^{2}-\left\langle\left|v_{q}\right|^{2}\right\rangle\right)$ is, as a sum of independent random variables, normal distributed. Note that only a prefactor of $1 / \sqrt{N^{d}}$ is required for that 
property. In particular, if $F$ is some bounded function independent of $N$, sums which come with a prefactor of $1 / N^{d}$ like $\frac{1}{N^{d}} \sum_{q} c_{q}\left|v_{q}\right|^{2}$ can be substituted by their expectation value,

$$
\lim _{N \rightarrow \infty} \int F\left(\frac{1}{N^{d}} \sum_{k} c_{k}\left|v_{k}\right|^{2}\right) d P(v)=F\left(\lim _{N \rightarrow \infty} \frac{1}{N^{d}} \sum_{k} c_{k}\left\langle\left|v_{k}\right|^{2}\right\rangle\right)
$$

Therefore, in the two loop approximation, one obtains in the infinite volume limit

$$
\langle G\rangle(k)=\frac{1}{a_{k}-\frac{\lambda^{2}}{N^{d}} \sum_{p} \frac{\left\langle\left|v_{k-p}\right|^{2}\right\rangle}{a_{p}-\frac{\lambda^{2}}{N^{d}} \sum_{t} \frac{\left\langle\left|v_{p-t}\right|^{2}\right\rangle}{a_{t}-\frac{\lambda^{2}}{N^{d}} \Sigma \cdots}}}=: \frac{1}{a_{k}-\left\langle\sigma_{k}\right\rangle}
$$

where the quantity $\left\langle\sigma_{k}\right\rangle$ satisfies the integral equation

$$
\left\langle\sigma_{k}\right\rangle=\frac{\lambda^{2}}{N^{d}} \sum_{p} \frac{\left\langle\left|v_{k-p}\right|^{2}\right\rangle}{a_{p}-\left\langle\sigma_{p}\right\rangle} \stackrel{L \rightarrow \infty}{\rightarrow} \frac{\lambda^{2}}{M^{d}} \int_{[0,2 \pi M]^{d}} \frac{d^{d} p}{(2 \pi)^{d}} \frac{\left\langle\left|v_{k-p}\right|^{2}\right\rangle}{a_{p}-\left\langle\sigma_{p}\right\rangle}
$$

For a Gaussian distribution $\left\langle\left|v_{q}\right|^{2}\right\rangle=1$ for all $q$ such that $\left\langle\sigma_{k}\right\rangle=\langle\sigma\rangle$ becomes independent of $k$. Thus we end up with

$$
\langle G\rangle(k)=\frac{1}{4 M^{2} \sum_{i=1}^{d} \sin ^{2}\left[\frac{k_{i}}{2 M}\right]-E-i \varepsilon-\langle\sigma\rangle}
$$

where $\langle\sigma\rangle$ is a solution of

$$
\begin{aligned}
\langle\sigma\rangle & =\frac{\lambda^{2}}{M^{d}} \int_{[0,2 \pi M]^{d}} \frac{d^{d} p}{(2 \pi)^{d}} \frac{1}{4 M^{2} \sum_{i=1}^{d} \sin ^{2}\left[\frac{p_{i}}{2 M}\right]-z-\langle\sigma\rangle} \\
& =\lambda^{2} \int_{[0,2 \pi]^{d}} \frac{d^{d} p}{(2 \pi)^{d}} \frac{1}{4 M^{2} \sum_{i=1}^{d} \sin ^{2}\left[\frac{p_{i}}{2}\right]-z-\langle\sigma\rangle} .
\end{aligned}
$$

This equation is of course well known and one deduces from it that it generates a small imaginary part $\operatorname{Im} \sigma=O\left(\lambda^{2}\right)$ if the energy $E$ is within the spectrum of $-\Delta$.

We now add the higher loop terms (the terms for $r>2$ in the denominator of (1.3)) to our discussion and give an interpretation in terms of Feynman diagrams. To make the volume factors more explicit, asume that the lattice spacing in coordinate space is $1 / M=1$ such that $N=L$.

For the Anderson model, Feynman graphs may be obtained by brutally expanding

$$
\begin{aligned}
& \int\left[a_{k} \delta_{k, p}+\frac{\lambda}{\sqrt{L}^{d}} v_{k-p}\right]_{k, k}^{-1} d P=\sum_{r=0}^{\infty} \int\left(C[V C]^{r}\right)_{k k} d P \\
& =\sum_{r=0}^{\infty} \frac{(-\lambda)^{r}}{{\sqrt{d^{d}}}^{r}} \sum_{p_{2} \cdots p_{r}} \frac{1}{a_{k} a_{p_{2}} \cdots a_{p_{r}} a_{k}} \int v_{k-p_{2}} v_{p_{2}-p_{3}} \cdots v_{p_{r}-k} d P
\end{aligned}
$$




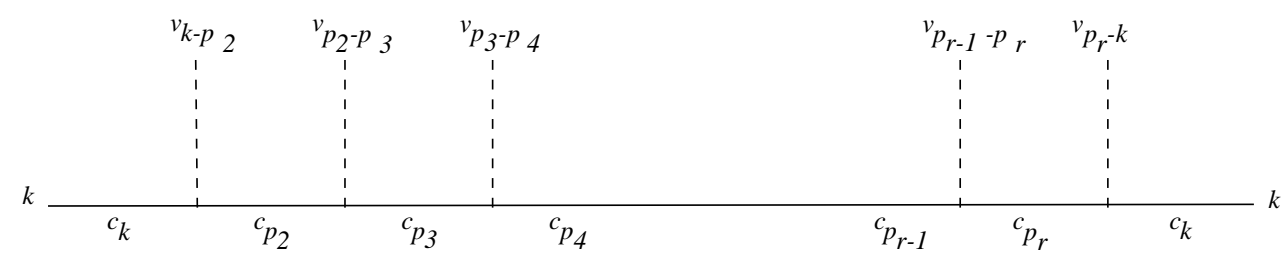

Figure 1: A string of particle lines with unpaired squiggles (dashed lines)

For a given $r$, this may be represented as in figure $1\left(c_{k}:=1 / a_{k}\right)$.

The integral over the $v$ gives a sum of $(r-1) ! !$ terms where each term is a product of $r / 2$ Kroenecker-delta's, the terms for odd $r$ vanish. If this is substituted in (2.24), the number of independent momentum sums is cut down to $r / 2$ and each of the $(r-1)$ !! terms may be represented by a diagram

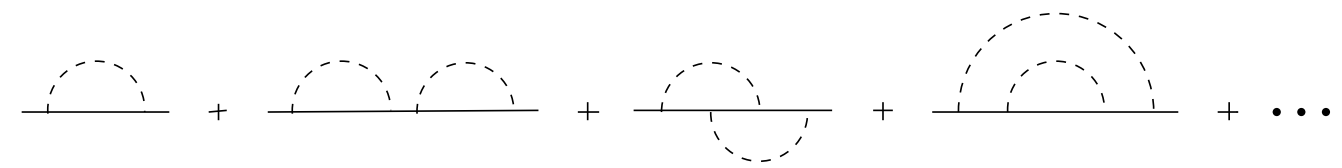

Figure 2: Lowest order diagrams contributing to (2.24) for $r=2$ and $r=4$

where, say, the value of the third diagram is given by $\frac{\lambda^{4}}{L^{2 d}} \sum_{p_{1}, p_{2}} \frac{1}{a_{k} a_{k+p_{1}} a_{k+p_{1}+p_{2}} a_{k+p_{2}} a_{k}}$. For short:

$$
\langle G\rangle(k)=\text { sum of all two legged diagrams . }
$$

Since the value of a diagram depends on its subgraph structure, one distinguishes, in the easiest case, two types of diagrams. Diagrams with or without two-legged subdiagrams. Those diagrams with two-legged subgraphs usually produce anomalously large contributions. They are devided further into the one-particle irreducible ones and the reducible ones. Thereby a diagram is called one-particle reducible if it can be made disconnected by cutting one solid or 'particle' line (no squiggle or dashed line), see also figure 3.

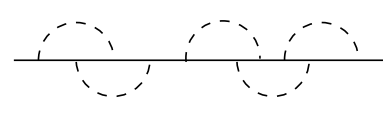

(a) reducible

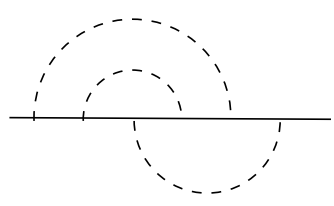

(b) irrdeducible, no two-legged subgraphs

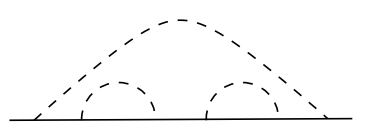

(c) irreducible, with two-legged
subgraphs

Figure 3: Reducible and irreducible diagrams

The reason for introducing reducible and irreducible diagrams is that the reducible ones can be easily resummed by writing down the Schwinger-Dyson equation which states that 
if the self energy $\Sigma_{k}$ is defined through

$$
\langle G\rangle(k)=\frac{1}{a_{k}-\Sigma_{k}\left(G_{0}\right)}
$$

then $\Sigma_{k}\left(G_{0}\right)$ is the sum of all amputated (no $1 / a_{k}$ 's at the ends) one particle irreducible diagrams. Here we wrote $\Sigma_{k}\left(G_{0}\right)$ to indicate that the factors ('propagators') assigned to the solid lines of the diagrams contributing to $\Sigma_{k}$ are given by the free two point function $G_{0}(p)=\frac{1}{a_{p}}$. However, the diagrams contributing to $\Sigma_{k}\left(G_{0}\right)$ still contain anomalously large contributions, namely irreducible graphs which contain two-legged subgraphs like diagram (c) in figure 3.

In the following we show, using the inversion formula (1.3) including all higher loop terms, that all graphs with two-legged subgraphs can be eliminated or resummed by writing down the following integral equation for $\langle G\rangle$ :

$$
\langle G\rangle(k)=\frac{1}{a_{k}-\sigma_{k}(\langle G\rangle)}
$$

where

$\sigma_{k}(\langle G\rangle)$ is the sum of all amputated two-legged diagrams which do not contain any two-legged subdiagrams, but now with propagators $\langle G\rangle(k)=\frac{1}{a_{k}-\sigma_{k}}$ instead of $G_{0}=\frac{1}{a_{k}}$

which may be formalized as in (2.35) below. The advantage of this formula is that the series for $\sigma_{k}(\langle G\rangle)$ can be expected to be asymptotic, that is, its lowest order contributions are a good approximation if the coupling is small, but, usually, the series for $\Sigma_{k}\left(G_{0}\right)$ is not asymptotic. Thus, in order to rigorously controll $\langle G\rangle(k)$, one has to define a suitable space of propagators, to estimate the sum of all amputated two-legged graphs without two-legged subgraphs on that space and then finally to show that the equation (2.27) has a solution on this space. We intend to address this problem in another paper.

We now show (2.27) for the Anderson model. For fixed $v$ one has

$$
G(k, v)=\frac{1}{a_{k}-\sigma_{k}(v)}
$$

where

$$
\sigma_{k}(v)=\sum_{r=2}^{L^{d}}(-1)^{r} \sum_{\substack{p_{2} \cdots p_{r} \\ p_{i} \neq p_{j}, p_{i} \neq k}} \frac{\lambda^{r}}{{\sqrt{L^{d}}}^{r}} G_{k}\left(p_{2}\right) \cdots G_{k p_{2} \cdots p_{r-1}}\left(p_{r}\right) v_{k-p_{2}} \cdots v_{p_{r}-k}
$$

We cutoff the $r$-sum in (2.29) at some arbitrary but fixed order $\ell<L^{d}$ where $\ell$ is choosen to be independent of the volume. Furthermore we substitute $G_{k p_{2} \cdots p_{j}}(p)$ by $G(p)$. Thus

$$
\langle G\rangle(k)=\left\langle\frac{1}{a_{k}-\sum_{r=2}^{\ell} \sigma_{k}^{r}(v)}\right\rangle
$$


where

$$
\sigma_{k}^{r}(v)=(-1)^{r} \frac{\lambda^{r}}{\sqrt{L^{d}}} \sum_{\substack{p_{2} \cdots p_{r} \\ p_{i} \neq p_{j}, p_{i} \neq k}} G\left(p_{2}\right) \cdots G\left(p_{r}\right) v_{k-p_{2}} \cdots v_{p_{r}-k}
$$

Consider first two strings $s_{k}^{r_{1}}, s_{k}^{r_{2}}$ where

$$
s_{k}^{r}(v)=\frac{\lambda^{r}}{\sqrt{L^{d}}} \sum_{\substack{p_{2} \cdots p_{r} \\ p_{i} \neq p_{j}, p_{i} \neq k}} c_{k p_{2} \cdots p_{r}}^{r} v_{k-p_{2}} \cdots v_{p_{r}-k}
$$

and the $c_{k p_{2} \cdots p_{r}}^{r}$ are some numbers. Then in the infinite volume limit

$$
\left\langle s_{k}^{r_{1}} s_{k}^{r_{2}}\right\rangle=\left\langle s_{k}^{r_{1}}\right\rangle\left\langle s_{k}^{r_{2}}\right\rangle
$$

because all pairings which connect the two strings have an extra volume factor $1 / L^{d}$. Namely, if the two strings are disconnected, there are $\left(r_{1}+r_{2}\right) / 2$ loops and a volume factor of $1 /{\sqrt{L^{d}}}^{\left(r_{1}+r_{2}\right)}$ giving $\left(r_{1}+r_{2}\right) / 2$ Riemannian sums. If the two strings are connected, there are only $\left(r_{1}+r_{2}-2\right) / 2$ loops leaving an extra factor of $1 / L^{d}$. By the same argument one has in the infinite volume limit

$$
\left\langle\left(s_{k}^{r_{1}}\right)^{n_{1}} \cdots\left(s_{k}^{r_{m}}\right)^{n_{m}}\right\rangle=\left\langle s_{k}^{r_{1}}\right\rangle^{n_{1}} \cdots\left\langle s_{k}^{r_{m}}\right\rangle^{n_{m}}
$$

which results in

$$
\langle G\rangle(k)=\frac{1}{a_{k}-\sum_{r=2}^{\ell} \frac{(-\lambda)^{r}}{{\sqrt{L^{d}}}^{r}} \sum_{\substack{p_{2} \cdots p_{r} \\ p_{i} \neq p_{j}, p_{i} \neq k}}\langle G\rangle\left(p_{2}\right) \cdots\langle G\rangle\left(p_{r}\right)\left\langle v_{k-p_{2}} \cdots v_{p_{r}-k}\right\rangle}
$$

The condition $p_{2}, \cdots, p_{r} \neq k$ and $p_{i} \neq p_{j}$ means exactly that two-legged subgraphs are forbidden. Namely, for a two-legged subdiagram as in (c) in figure 3, the incomming and outgoing momenta $p, p^{\prime}$ (to which are assigned propagators $\langle G\rangle(p),\langle G\rangle\left(p^{\prime}\right)$ ) must be equal which is forbidden by the condidtion $p_{i} \neq p_{j}$ in $(2.35)$.

However, we cannot take the limit $\ell \rightarrow \infty$ in (2.35) since the series in the denominator of (2.35) is only an asymptotic one. To see this a bit more clearly suppose for the moment that there were no restrictions on the momentum sums. Then, if $V=\left(\frac{\lambda}{\sqrt{L^{d}}} v_{k-p}\right)_{k, p}$ and $\langle G\rangle=\left(\langle G\rangle(k) \delta_{k, p}\right)_{k, p}$

$$
\frac{\lambda^{r}}{\sqrt{L^{d^{r}}}} \sum_{p_{2} \cdots p_{r}}\langle G\rangle\left(p_{2}\right) \cdots\langle G\rangle\left(p_{r}\right)\left\langle v_{k-p_{2}} \cdots v_{p_{r}-k}\right\rangle=\left\langle\left(V[\langle G\rangle V]^{r-1}\right)_{k k}\right\rangle
$$

and for $\ell \rightarrow \infty$ we would get

$$
\langle G\rangle(k)=\frac{1}{a_{k}-\left\langle\left(V \frac{\langle G\rangle V}{I d+\langle G\rangle V}\right)_{k k}\right\rangle}=\frac{1}{a_{k}-\left\langle\left(V \frac{1}{\langle G\rangle^{-1}+V} V\right)_{k k}\right\rangle}
$$


That is, the factorials produced by the number of diagrams in the denominator of (2.35) are basically the same as those in the expansion

$$
\int_{\mathbb{R}} \frac{x^{2}}{z+\lambda x} e^{-\frac{x^{2}}{2}} \frac{d x}{\sqrt{2 \pi}}=\sum_{r=0}^{\ell} \frac{\lambda^{2 r}}{z^{2 r+1}}(2 r+1) ! !+R_{\ell+1}(\lambda)
$$

where the remainder satisfies the bound $\left|R_{\ell+1}(\lambda)\right| \leq \ell$ ! $\operatorname{const}_{z}^{\ell} \lambda^{\ell}$.

We close this section with two further remarks. So far the computations were done in momentum space. One may wonder what one gets if the inversion formula (1.3) is applied to $[-\Delta+z+\lambda V]^{-1}$ in coordinate space. Whereas a geometric series expansion of $[-\Delta+z+\lambda V]^{-1}$ gives a representation in terms of the simple random walk, application of (1.3) results in a representation in terms of the self avoiding walk:

$$
[-\Delta+z+\lambda V]_{0, x}^{-1}=\sum_{\substack{\gamma: 0 \rightarrow x \\ \gamma \text { self avoiding }}} \frac{\operatorname{det}\left[(-\Delta+z+\lambda V)_{y, y^{\prime} \in \Gamma \backslash \gamma}\right]}{\operatorname{det}\left[(-\Delta+z+\lambda V)_{y, y^{\prime} \in \Gamma}\right]}
$$

where $\Gamma$ is the lattice in coordinate space. Namely, if $|x|>1$, the inversion formula (1.4) for the off-diagonal elements gives

$$
\begin{aligned}
& {[-\Delta+\lambda V]_{0, x}^{-1}=\sum_{r=3}^{L^{d}}(-1)^{r+1} \sum_{\substack{x_{3} \cdots x_{r} \in \Gamma \backslash\{0, x\} \\
x_{i} \neq x_{j}}} G(0) G_{0}\left(x_{3}\right) \cdots G_{0 x_{3} \cdots x_{r}}(x)(-\Delta)_{0 x_{3}} \cdots(-\Delta)_{x_{r} x}} \\
& \quad=\sum_{r=3}^{L^{d}} \sum_{\substack{x_{2}=0, x_{3}, \cdots, x_{r}, x_{r+1}=x \in \Gamma \\
\left|x_{i}-x_{i+1}\right|=1 \forall i=2 \cdots r}} \frac{\operatorname{det}\left[(-\Delta+\lambda V)_{y, y^{\prime} \in \Gamma \backslash\{0\}}\right]}{\operatorname{det}\left[(-\Delta+\lambda V)_{\left.y, y^{\prime} \in \Gamma\right]}\right]} \cdots \frac{\operatorname{det}\left[(-\Delta+\lambda V)_{y, y^{\prime} \in \Gamma \backslash\left\{0, x_{3} \cdots x_{r}, x\right\}}\right]}{\operatorname{det}\left[(-\Delta+\lambda V)_{y, y^{\prime} \in \Gamma \backslash\left\{0, x_{3} \cdots x_{r}\right\}}\right]}
\end{aligned}
$$

which coincides with (2.39).

Finally we remark that, while the argument following (2.32) leads to a factorization property for on-diagonal elements in momentum space, $\langle G(k) G(p)\rangle=\langle G(k)\rangle\langle G(p)\rangle$, there is no such property for products of off-diagonal elements which appear in a quantity like

$$
\Lambda(q)=\frac{1}{L^{d}} \sum_{k, p}\left\langle\left[a_{k} \delta_{k, p}+\frac{\lambda}{\sqrt{L}} v_{k-p}\right]_{k, p}^{-1}\left[\bar{a}_{k} \delta_{k, p}+\frac{\lambda}{\sqrt{L}} \bar{v}_{k-p}\right]_{k-q, p-q}^{-1}\right\rangle
$$

which is the Fourier transform of $\left\langle\left|[-\Delta+z+\lambda V]_{x, y}^{-1}\right|^{2}\right\rangle$. (Each off-diagonal inverse matrix element is proportional to $1 / \sqrt{L^{d}}$, therefore the prefactor of $1 / L^{d}$ in (2.40) is correct.) 


\section{Proof of the Inversion Formula}

Lemma 3.1: Let $B \in \mathbb{C}^{k \times n}, C \in \mathbb{C}^{n \times k}$ and let $I d_{k}$ denote the identity in $\mathbb{C}^{k \times k}$. Then:

(i) $I d_{k}-B C$ invertible $\Leftrightarrow I d_{n}-C B$ invertible.

(ii) If the left or the right hand side of (i) fullfilled, then $C \frac{1}{I d_{k}-B C}=\frac{1}{I d_{n}-C B} C$.

Proof: Let

$$
B=\left(\begin{array}{ccc}
- & \vec{b}_{1} & - \\
\vdots & \\
- & \overrightarrow{b_{k}} & -
\end{array}\right), \quad C=\left(\begin{array}{ccc}
\mid & & \mid \\
\vec{c}_{1} & \cdots & \vec{c}_{k} \\
\mid & & \mid
\end{array}\right)
$$

where the $\vec{b}_{j}$ are $n$-component row vectors and the $\vec{c}_{j}$ are $n$-component column vectors. Let $\vec{x} \in \operatorname{Kern}(I d-C B)$. Then $\vec{x}=C B \vec{x}=\sum_{j} \lambda_{j} \vec{c}_{j}$ if we define $\lambda_{j}:=\left(\vec{b}_{j}, \vec{x}\right)$. Let $\vec{\lambda}=\left(\lambda_{j}\right)_{1 \leq j \leq k}$. Then $[(I d-B C) \vec{\lambda}]_{i}=\lambda_{i}-\sum_{j}\left(\vec{b}_{i}, \vec{c}_{j}\right) \lambda_{j}=\left(\vec{b}_{i}, \vec{x}\right)-\sum_{j}\left(\vec{b}_{i}, \vec{c}_{j}\right) \lambda_{j}=0$ since $\vec{x}=\sum_{j} \lambda_{j} \vec{c}_{j}$, thus $\vec{\lambda} \in \operatorname{Kern}(I d-B C)$. On the other hand, if some $\vec{\lambda} \in \operatorname{Kern}(I d-B C)$, then $\vec{x}:=\sum_{j} \lambda_{j} \vec{c}_{j} \in \operatorname{Kern}(I d-C B)$ which proves (i). Part (ii) then follows from $C=$ $\frac{1}{I d_{n}-C B}\left(I d_{n}-C B\right) C=\frac{1}{I d_{n}-C B} C\left(I d_{k}-B C\right)$

The inversion formula $(1.3,4)$ is obtained by iterative application of the next lemma, which states the Feshbach formula for finite dimensional matrices. For a more general version one may look in [BFS], Theorem 2.1.

Lemma 3.2: Let $h=\left(\begin{array}{ll}A & B \\ C & D\end{array}\right) \in \mathbb{C}^{n \times n}$ where $A \in \mathbb{C}^{k \times k}, D \in \mathbb{C}^{(n-k) \times(n-k)}$ are invertible and $B \in \mathbb{C}^{k \times(n-k)}, C \in \mathbb{C}^{(n-k) \times k}$. Then

$$
h \text { invertible } \Leftrightarrow A-B D^{-1} C \text { invertible } \Leftrightarrow D-C A^{-1} B \text { invertible }
$$

and if one of the conditions in (3.1) is fullfilled, one has $h^{-1}=\left(\begin{array}{ll}E & F \\ G & H\end{array}\right)$ where

$$
\begin{aligned}
E & =\frac{1}{A-B D^{-1} C}, & H & =\frac{1}{D-C A^{-1} B}, \\
F=-E B D^{-1} & =-A^{-1} B H, & & =-H C A^{-1}=-D^{-1} C E .
\end{aligned}
$$

Proof: We have, using Lemma 3.1 in the second line,

$$
\begin{aligned}
A-B D^{-1} C \text { inv. } & \Leftrightarrow I d_{k}-A^{-1} B D^{-1} C \text { inv. } \\
& \Leftrightarrow I d_{n-k}-D^{-1} C A^{-1} B \text { inv. } \\
& \Leftrightarrow D-C A^{-1} B \text { inv. }
\end{aligned}
$$


Furthermore, again by Lemma 3.1,

$$
D^{-1} C \frac{1}{I d-A^{-1} B D^{-1} C}=\frac{1}{I d-D^{-1} C A^{-1} B} D^{-1} C=\frac{1}{D-C A^{-1} B} C=H C
$$

and

$$
A^{-1} B \frac{1}{I d-D^{-1} C A^{-1} B}=\frac{1}{I d-A^{-1} B D^{-1} C} A^{-1} B=\frac{1}{A-B D^{-1} C} B=E B
$$

which proves the last equalities in (3.3), $H C A^{-1}=D^{-1} C E$ and $E B D^{-1}=A^{-1} B H$. Using these equations and the definition of $E, F, G$ and $H$ one computes

$$
\left(\begin{array}{ll}
A & B \\
C & D
\end{array}\right)\left(\begin{array}{ll}
E & F \\
G & H
\end{array}\right)=\left(\begin{array}{ll}
E & F \\
G & H
\end{array}\right)\left(\begin{array}{ll}
A & B \\
C & D
\end{array}\right)=\left(\begin{array}{cc}
I d & 0 \\
0 & I d
\end{array}\right)
$$

It remains to show that the invertibility of $h$ implies the invertibility of $A-B D^{-1} C$. To this end let $P=\left(\begin{array}{cc}I d & \\ & 0\end{array}\right), \bar{P}=\left(\begin{array}{ll}{ }^{0} & \\ & I d\end{array}\right)$ such that $A-B D^{-1} C=P h P-P h \bar{P}(\bar{P} h \bar{P})^{-1} \bar{P} h P$. Then

$$
\begin{aligned}
\left(A-B D^{-1} C\right) P h^{-1} P & =P h P h^{-1} P-P h \bar{P}(\bar{P} h \bar{P})^{-1} \bar{P} h P h^{-1} P \\
& =P h(1-\bar{P}) h^{-1} P-P h \bar{P}(\bar{P} h \bar{P})^{-1} \bar{P} h(1-\bar{P}) h^{-1} P \\
& =P-P h \bar{P} h^{-1} P+P h \bar{P} h^{-1} P=P
\end{aligned}
$$

and similarly $P h^{-1} P\left(A-B D^{-1} C\right)=P$ which proves the invertibility of $A-B D^{-1} C$

Theorem 3.3: Let $B \in \mathbb{C}^{n N \times n N}$ be given by $B=\left(B_{k p}\right)_{k, p \in \mathcal{M}}, \mathcal{M}$ some index set, $|\mathcal{M}|=N$, and $B_{k p}=\left(B_{k \sigma, p \tau}\right)_{\sigma, \tau \in I} \in \mathbb{C}^{n \times n}$ where $I$ is another index set, $|I|=n$. Suppose that $B$ and, for any $\mathcal{N} \subset \mathcal{M}$, the submatrix $\left(B_{k p}\right)_{k, p \in \mathcal{N}}$ is invertible. For $k \in \mathcal{M}$ let

$$
G(k):=\left[B^{-1}\right]_{k k} \in \mathbb{C}^{n \times n}
$$

and, if $\mathcal{N} \subset \mathcal{M}, k \notin \mathcal{N}$,

$$
G_{\mathcal{N}}(k):=\left[\left\{\left(B_{s t}\right)_{s, t \in \mathcal{M} \backslash \mathcal{N}}\right\}^{-1}\right]_{k k} \in \mathbb{C}^{n \times n}
$$

Then one has

(i) The on-diagonal block matrices of $B^{-1}$ are given by

$$
G(k)=\frac{1}{B_{k k}-\sum_{r=2}^{N}(-1)^{r} \sum_{\substack{p_{2} \cdots p_{r} \in \mathcal{M} \backslash\{k\} \\ p_{i} \neq p_{j}}} B_{k p_{2}} G_{k}\left(p_{2}\right) B_{p_{2} p_{3}} \cdots B_{p_{r-1} p_{r}} G_{k p_{2} \cdots p_{r-1}}\left(p_{r}\right) B_{p_{r} k}}
$$

where $1 / \cdot$ is inversion of $n \times n$ matrices. 
(ii) Let $k, p \in \mathcal{M}, k \neq p$. Then the off-diagonal block matrices of $B^{-1}$ can be expressed in terms of the $G_{\mathcal{N}}(s)$ and the $B_{s t}$,

$$
\left[B^{-1}\right]_{k p}=-G(k) B_{k p} G_{k}(p)-\sum_{r=3}^{N}(-1)^{r} \sum_{\substack{t_{3} \cdots t_{r} \in \mathcal{M} \backslash\{k, p\} \\ t_{i} \neq t_{j}}} G(k) B_{k t_{3}} G_{k}\left(t_{3}\right) B_{t_{3} t_{4}} \cdots B_{t_{r} p} G_{k t_{3} \cdots t_{r}}(p)
$$

Proof: Let $k$ be fixed and let $p, p^{\prime} \in \mathcal{M} \backslash\{k\}$ below label columns and rows. By Lemma 3.2 we have

$$
\left(\begin{array}{cc}
G(k) & \\
& *
\end{array}\right)=\left(\begin{array}{ccc}
B_{k k} & - & B_{k p} \\
\mid & & \\
B_{p^{\prime} k} & B_{p^{\prime} p} \\
\mid & &
\end{array}\right)^{-1}=\left(\begin{array}{ccc}
E & -F- \\
\mid & H \\
G & H
\end{array}\right)
$$

where

$$
\begin{aligned}
G(k)=E & =\frac{1}{B_{k k}-\sum_{p, p^{\prime} \neq k} B_{k p}\left[\left\{\left(B_{p^{\prime} p}\right)_{p^{\prime}, p \in \mathcal{M} \backslash\{k\}}\right\}^{-1}\right]_{p p^{\prime}} B_{p^{\prime} k}} \\
& =\frac{1}{B_{k k}-\sum_{p \neq k} B_{k p} G_{k}(p) B_{p k}-\sum_{\substack{p, p^{\prime} \neq k \\
p \neq p^{\prime}}} B_{k p}\left[\left\{\left(B_{p^{\prime} p}\right)_{p^{\prime}, p \in \mathcal{M} \backslash\{k\}}\right\}^{-1}\right]_{p p^{\prime}} B_{p^{\prime} k}}
\end{aligned}
$$

and

$$
\begin{aligned}
F_{k p}=\left[B^{-1}\right]_{k p} & =-G(k) \sum_{t \neq k} B_{k t}\left[\left\{\left(B_{p^{\prime} p}\right)_{p^{\prime}, p \in \mathcal{M} \backslash\{k\}}\right\}^{-1}\right]_{t p} \\
& =-G(k) B_{k p} G_{k}(p)-G(k) \sum_{t \neq k, p} B_{k t}\left[\left\{\left(B_{p^{\prime} p}\right)_{p^{\prime}, p \in \mathcal{M} \backslash\{k\}}\right\}^{-1}\right]_{t p}
\end{aligned}
$$

Apply Lemma 3.2 now to the matrix $\left\{\left(B_{p^{\prime} p}\right)_{p^{\prime}, p \in \mathcal{M} \backslash\{k\}}\right\}^{-1}$ and proceed by induction to obtain after $\ell$ steps

$$
\begin{aligned}
& G(k)= \frac{1}{B_{k k}-\sum_{r=2}^{\ell}(-1)^{r} \sum_{\substack{p_{2} \cdots p_{r} \in \mathcal{M} \backslash\{k\} \\
p_{i} \neq p_{j}}} B_{k p_{2}} G_{k}\left(p_{2}\right) B_{p_{2} p_{3}} \cdots B_{p_{r-1} p_{r}} G_{k p_{2} \cdots p_{r-1}}\left(p_{r}\right) B_{p_{r} k}-R_{\ell+1}} \\
& F_{k p}=-G(k) B_{k p} G_{k}(p)-\sum_{r=3}^{\ell}(-1)^{r} \sum_{\substack{t_{3} \cdots t_{r} \in \mathcal{M} \backslash\{k, p\} \\
t_{i} \neq t_{j}}} G(k) B_{k t_{3}} G_{k}\left(t_{3}\right) B_{t_{3} t_{4}} \cdots B_{t_{r} p} G_{k t_{3} \cdots t_{r}}(p)-\tilde{R}_{\ell+1}
\end{aligned}
$$


where

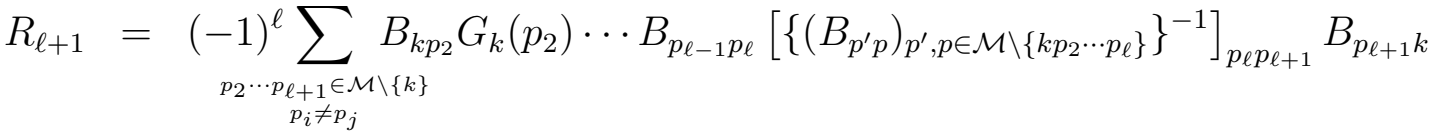

$$
\begin{aligned}
& \tilde{R}_{\ell+1}=(-1)^{\ell} \sum_{\substack{t_{3} \cdots t_{\ell+1} \in \mathcal{M} \backslash\{k, p\} \\
t_{i} \neq t_{j}}} G(k) B_{k t_{3}} \cdots G_{k t_{3} \cdots t_{\ell-1}}\left(t_{\ell}\right) B_{t_{\ell} t_{\ell+1}}\left[\left\{\left(B_{p^{\prime} p}\right)_{p^{\prime}, p \in \mathcal{M} \backslash\left\{k t_{3} \cdots t_{\ell}\right\}}\right\}^{-1}\right]_{t_{\ell+1} p}
\end{aligned}
$$

Since $R_{N+1}=\tilde{R}_{N+1}=0$ the theorem follows

\section{Application to the Many-Electron System and to the $\varphi^{4}$-Model}

\subsection{The Many-Electron System}

We consider the many-electron system in the grand canonical ensemble in finite volume $[0, L]^{d}$ and at some small but positive temperature $T=1 / \beta>0$ with attractive deltainteraction given by the Hamiltonian

$$
H=H_{0}-\lambda H_{\mathrm{int}}=\frac{1}{L^{d}} \sum_{\mathbf{k} \sigma}\left(\frac{\mathbf{k}^{2}}{2 m}-\mu\right) a_{\mathbf{k} \sigma}^{+} a_{\mathbf{k} \sigma}-\frac{\lambda}{L^{3 d}} \sum_{\mathbf{k p q}} a_{\mathbf{k} \uparrow}^{+} a_{\mathbf{q}-\mathbf{k} \downarrow}^{+} a_{\mathbf{q}-\mathbf{p} \downarrow} a_{\mathbf{p} \uparrow}
$$

Our normalization conventions concerning the volume factors are such that the canonical anticommutation relations read $\left\{a_{\mathbf{k} \sigma}, a_{\mathbf{p} \tau}^{+}\right\}=L^{d} \delta_{\mathbf{k}, \mathbf{p}} \delta_{\sigma, \tau}$. The momentum sums range

over some subset of $\left(\frac{2 \pi}{L} \mathbb{Z}\right)^{d}$, say $\mathcal{M}=\left\{\mathbf{k} \in\left(\frac{2 \pi}{L} \mathbb{Z}\right)^{d}|| e_{\mathbf{k}} \mid \leq 1\right\}, e_{\mathbf{k}}=\mathbf{k}^{2} / 2 m-\mu$, and $\mathbf{q} \in\{\mathbf{k}-\mathbf{p} \mid \mathbf{k}, \mathbf{p} \in \mathcal{M}\}$.

We are interested in the momentum distribution

$$
\left\langle a_{\mathbf{k} \sigma}^{+} a_{\mathbf{k} \sigma}\right\rangle=\operatorname{Tr}\left[e^{-\beta H} a_{\mathbf{k} \sigma}^{+} a_{\mathbf{k} \sigma}\right] / \operatorname{Tr} e^{-\beta H}
$$

and in the expectation value of the energy

$$
\left\langle H_{\text {int }}\right\rangle=\sum_{\mathbf{q}} \Lambda(\mathbf{q})
$$


where

$$
\Lambda(\mathbf{q})=\frac{\lambda}{L^{3 d}} \sum_{\mathbf{k}, \mathbf{p}} \operatorname{Tr}\left[e^{-\beta H} a_{\mathbf{k} \uparrow}^{+} a_{\mathbf{q}-\mathbf{k} \downarrow}^{+} a_{\mathbf{q}-\mathbf{p} \downarrow} a_{\mathbf{p} \uparrow}\right] / \operatorname{Tr} e^{-\beta H}
$$

By writing down the perturbation series for the partition function, rewriting it as a Grassmann integral

$$
\begin{aligned}
\frac{\operatorname{Tr} e^{-\beta\left(H_{0}-\lambda H_{\text {int }}\right)}}{\operatorname{Tr} e^{-\beta H_{0}}} & =\int e^{\frac{\lambda}{\left(\beta L^{d}\right)^{3}} \sum_{k p q} \bar{\psi}_{k \uparrow} \bar{\psi}_{q-k \downarrow} \psi_{q-p \downarrow} \psi_{p \uparrow}} d \mu_{C}(\psi, \bar{\psi}) \\
d \mu_{C} & =\prod_{k \sigma} \frac{\beta L^{d}}{i k_{0}-e_{\mathbf{k}}} e^{-\frac{1}{\beta L^{d}} \sum_{k \sigma}\left(i k_{0}-e_{\mathbf{k}}\right) \bar{\psi}_{k \sigma} \psi_{k \sigma}} \prod_{k \sigma} d \psi_{k \sigma} d \bar{\psi}_{k \sigma},
\end{aligned}
$$

performing a Hubbard-Stratonovich transformation $\left(\phi_{q}=u_{q}+i v_{q}, d \phi_{q} d \bar{\phi}_{q}:=d u_{q} d v_{q}\right)$

$$
e^{-\sum_{q} a_{q} b_{q}}=\int e^{i \sum_{q}\left(a_{q} \phi_{q}+b_{q} \bar{\phi}_{q}\right)} e^{-\sum_{q}\left|\phi_{q}\right|^{2}} \prod_{q} \frac{d \phi_{q} d \bar{\phi}_{q}}{\pi}
$$

with

$$
a_{q}=\frac{\lambda^{\frac{1}{2}}}{\left(\beta L^{d}\right)^{\frac{3}{2}}} \sum_{k} \bar{\psi}_{k \uparrow} \bar{\psi}_{q-k \downarrow}, \quad b_{q}=\frac{\lambda^{\frac{1}{2}}}{\left(\beta L^{d}\right)^{\frac{3}{2}}} \sum_{p} \psi_{p \uparrow} \psi_{q-p \downarrow}
$$

and then integrating out the $\psi, \bar{\psi}$ variables, one arrives at the following representation which is the starting point for our analysis (for more details, see [FKT] or [L1]):

$$
\frac{1}{L^{d}}\left\langle a_{\mathbf{k} \sigma}^{+} a_{\mathbf{k} \sigma}\right\rangle=\frac{1}{\beta L^{d}} \frac{1}{\beta} \sum_{k_{0} \in \frac{\pi}{\beta}(2 \mathbb{Z}+1)}\left\langle\psi_{\mathbf{k} k_{0} \sigma}^{+} \psi_{\mathbf{k} k_{0} \sigma}\right\rangle
$$

where, abbreviating $k=\left(\mathbf{k}, k_{0}\right), \kappa=\beta L^{d}, a_{k}=i k_{0}-e_{\mathbf{k}}, g=\lambda^{\frac{1}{2}}$,

$$
\frac{1}{\kappa}\left\langle\bar{\psi}_{t \sigma} \psi_{t \sigma}\right\rangle=\int\left[\begin{array}{cc}
a_{k} \delta_{k, p} & \frac{i g}{\sqrt{\kappa}} \bar{\phi}_{p-k} \\
\frac{i g}{\sqrt{\kappa}} \phi_{k-p} & a_{-k} \delta_{k, p}
\end{array}\right]_{t \sigma, t \sigma}^{-1} d P(\phi)
$$

and $d P(\phi)$ is the normalized measure

$$
d P(\phi)=\frac{1}{Z} \operatorname{det}\left[\begin{array}{cc}
a_{k} \delta_{k, p} & \frac{i g}{\sqrt{\kappa}} \bar{\phi}_{p-k} \\
\frac{i g}{\sqrt{\kappa}} \phi_{k-p} & a_{-k} \delta_{k, p}
\end{array}\right] e^{-\sum_{q}\left|\phi_{q}\right|^{2}} \prod_{q} d \phi_{q} d \bar{\phi}_{q}
$$

Furthermore

$$
\Lambda(\mathbf{q})=\frac{1}{\beta} \sum_{q_{0} \in \frac{2 \pi}{\beta} \mathbb{Z}} \Lambda\left(\mathbf{q}, q_{0}\right)
$$

where

$$
\begin{aligned}
\Lambda(q) & =\frac{\lambda}{\left(\beta L^{d}\right)^{3}} \sum_{k, p}\left\langle\bar{\psi}_{k \uparrow} \bar{\psi}_{q-k \downarrow} \psi_{q-p \downarrow} \psi_{p \uparrow}\right\rangle \\
& =\left\langle\left|\phi_{q}\right|^{2}\right\rangle-1
\end{aligned}
$$


and the expectation in the last line is integration with respect to $d P(\phi)$. The expectation on the $\psi$ variables $\left\langle\bar{\psi}_{k \sigma} \psi_{k \sigma}\right\rangle=\frac{1}{\mathcal{Z}} \int \bar{\psi}_{k \sigma} \psi_{k \sigma} e^{\frac{\lambda}{\kappa^{3}} \sum_{k, p, q} \bar{\psi}_{k \uparrow} \bar{\psi}_{q-k \downarrow} \psi_{q-p \downarrow} \psi_{p \uparrow}} d \mu_{C}$ is Grassmann integration, but these representations are not used in the following. The matrix and the integral in (4.9) become finite dimensional if we choose some cutoff on the $k_{0}$ variables which is removed in the end. The set $\mathcal{M}$ for the spatial momenta is already finite since we have chosen a fixed UV-cuttoff $\left|e_{\mathbf{k}}\right|=\left|\mathbf{k}^{2} / 2 m-\mu\right| \leq 1$ which will not be removed in the end since we are interested in the infrared properties at $\mathbf{k}^{2} / 2 m=\mu$.

Our goal is to apply the inversion formula to the inverse matrix element in (4.9). Instead of writing the matrix in terms of four $N \times N$ blocks $\left(a_{k} \delta_{k, p}\right)_{k, p},\left(\bar{\phi}_{p-k}\right)_{k, p},\left(\phi_{k-p}\right)_{k, p}$ and $\left(a_{-k} \delta_{k, p}\right)_{k, p}$ where $N$ is the number of the $d+1$-dimensional momenta $k, p$, we interchange rows and columns to rewrite it in terms of $N$ blocks of size $2 \times 2$ (the matrix $U$ in the next line interchanges the rows and columns):

$$
U\left[\begin{array}{cc}
a_{k} \delta_{k, p} & \frac{i g}{\sqrt{\kappa}} \bar{\phi}_{p-k} \\
\frac{i g}{\sqrt{\kappa}} \phi_{k-p} & a_{-k} \delta_{k, p}
\end{array}\right] U^{-1}=B=\left(B_{k p}\right)_{k, p}
$$

where the $2 \times 2$ blocks $B_{k p}$ are given by

$$
B_{k k}=\left(\begin{array}{cc}
a_{k} & \frac{i g}{\sqrt{\kappa}} \bar{\phi}_{0} \\
\frac{i g}{\sqrt{\kappa}} \phi_{0} & a_{-k}
\end{array}\right), \quad B_{k p}=\frac{i g}{\sqrt{\kappa}}\left(\begin{array}{cc}
0 & \bar{\phi}_{p-k} \\
\phi_{k-p} & 0
\end{array}\right) \text { if } k \neq p .
$$

We want to compute the $2 \times 2$ matrix

$$
\langle G\rangle(k)=\int G(k) d P(\phi)
$$

where

$$
G(k)=\left[B^{-1}\right]_{k k}
$$

We start again with the two loop approximation which retains only the $r=2$ term in the denominator of (1.3). The result will be equation (4.20) below where the quantities $\left\langle\sigma_{k}\right\rangle$ and $\left\langle\left|\phi_{0}\right|^{2}\right\rangle$ appearing in (4.20) have to satisfy the equations (4.21) and (4.24) which have to be solved in conjunction with (4.29). The solution to these equations is discussed below (4.30).

We first derive (4.20). In the two loop approximation,

$$
\begin{aligned}
G(k) & \approx\left[B_{k k}-\sum_{p \neq k} B_{k p} G_{k}(p) B_{p k}\right]^{-1} \\
& =\left[\left(\begin{array}{cc}
a_{k} & \frac{i g}{\sqrt{\kappa}} \bar{\phi}_{0} \\
\frac{i g}{\sqrt{\kappa}} \phi_{0} & a_{-k}
\end{array}\right)+\frac{\lambda}{\kappa} \sum_{p \neq k}\left(\begin{array}{cc}
\phi_{k-p} & \bar{\phi}_{p-k} \\
\phi_{k-1}
\end{array}\right) G_{k}(p)\left(\begin{array}{cc}
\bar{\phi}_{k-p} \\
\phi_{p-k}
\end{array}\right)\right]^{-1} \\
& =:\left[\left(\begin{array}{cc}
a_{k} & \frac{i g}{\sqrt{\kappa}} \bar{\phi}_{0} \\
\frac{i g}{\sqrt{\kappa}} \phi_{0} & \bar{a}_{k}
\end{array}\right)+\Sigma(k)\right]^{-1}
\end{aligned}
$$


where, substituting again $G_{k}(p)$ by $G(p)$ in the infinite volume limit,

$$
\Sigma(k)=\frac{\lambda}{\kappa} \sum_{p \neq k}\left(\begin{array}{ll} 
& \bar{\phi}_{p-k} \\
\phi_{k-p} &
\end{array}\right)\left[\left(\begin{array}{cc}
a_{p} & \frac{i g}{\sqrt{\kappa}} \bar{\phi}_{0} \\
\frac{i g}{\sqrt{\kappa}} \phi_{0} & \bar{a}_{p}
\end{array}\right)+\Sigma(p)\right]^{-1}\left(\begin{array}{cc} 
& \bar{\phi}_{k-p} \\
\phi_{p-k} &
\end{array}\right)
$$

Anticipating the fact that the off-diagonal elements of $\langle\Sigma\rangle(k)$ will be zero (for 'zero external field'), we make the Ansatz

$$
\Sigma(k)=\left(\begin{array}{cc}
\sigma_{k} & \\
& \bar{\sigma}_{k}
\end{array}\right)
$$

and obtain

$$
\left(\begin{array}{cc}
\sigma_{k} & \\
& \bar{\sigma}_{k}
\end{array}\right)=\frac{\lambda}{\kappa} \sum_{p \neq k} \frac{1}{\left(a_{p}+\sigma_{p}\right)\left(\bar{a}_{p}+\bar{\sigma}_{p}\right)+\frac{\lambda}{\kappa}\left|\phi_{0}\right|^{2}}\left(\begin{array}{cc}
\left(a_{k}+\sigma_{k}\right)\left|\phi_{p-k}\right|^{2} & -\frac{i g}{\sqrt{\kappa}} \phi_{0} \bar{\phi}_{k-p} \bar{\phi}_{p-k} \\
-\frac{i g}{\sqrt{\kappa}} \bar{\phi}_{0} \phi_{k-p} \phi_{p-k} & \left(\bar{a}_{k}+\bar{\sigma}_{k}\right)\left|\phi_{k-p}\right|^{2}
\end{array}\right)
$$

As for the Anderson model, we perform the functional integral by substituting the quantities $\left|\phi_{q}\right|^{2}$ by their expectation values $\left\langle\left|\phi_{q}\right|^{2}\right\rangle$. Apparently this is less obvious in this case since $d P(\phi)$ is no longer Gaussian and the $\left|\phi_{q}\right|^{2}$ are no longer identically, independently distributed. We will comment on this after (4.37) below and at the end of the next section by reinterpreting this procedure as a resummation of diagrams. For now, we simply continue in this way. Then

$$
\langle G\rangle(k)=\frac{1}{\left|a_{k}+\left\langle\sigma_{k}\right\rangle\right|^{2}+\frac{\lambda}{\kappa}\left\langle\left|\phi_{0}\right|^{2}\right\rangle}\left(\begin{array}{cc}
\bar{a}_{k}+\left\langle\bar{\sigma}_{k}\right\rangle & -\frac{i g}{\sqrt{\kappa}}\left\langle\bar{\phi}_{0}\right\rangle \\
-\frac{i g}{\sqrt{\kappa}}\left\langle\phi_{0}\right\rangle & a_{k}+\left\langle\sigma_{k}\right\rangle
\end{array}\right)
$$

where the quantity $\left\langle\sigma_{k}\right\rangle$ has to satisfy the equation

$$
\left\langle\sigma_{k}\right\rangle=\frac{\lambda}{\kappa} \sum_{p \neq k} \frac{\bar{a}_{p}+\left\langle\bar{\sigma}_{p}\right\rangle}{\left|a_{p}+\left\langle\sigma_{p}\right\rangle\right|^{2}+\frac{\lambda}{\kappa}\left\langle\left|\phi_{0}\right|^{2}\right\rangle}\left\langle\left|\phi_{p-k}\right|^{2}\right\rangle
$$

Since $d P(\phi)$ is not Gaussian, we do not know the expectations $\left\langle\left|\phi_{q}\right|^{2}\right\rangle$. However, by partial integration, we obtain

$$
\left\langle\left|\phi_{q}\right|^{2}\right\rangle=1+\frac{i g}{\sqrt{\kappa}} \sum_{p} \int \phi_{q}\left[B^{-1}(\phi)\right]_{p \uparrow, p+q \downarrow} d P(\phi)
$$

Namely,

$$
\begin{aligned}
& \left\langle\left|\phi_{q}\right|^{2}\right\rangle=\frac{1}{Z} \int \phi_{q} \bar{\phi}_{q} \operatorname{det}\left[\left\{B_{k p}(\phi)\right\}_{k, p}\right] e^{-\sum_{q}\left|\phi_{q}\right|^{2}} d \phi_{q} d \bar{\phi}_{q} \\
& =1+\frac{1}{Z} \int \phi_{q}\left(\frac{\partial}{\partial \phi_{q}} \operatorname{det}\left[\left\{B_{k p}(\phi)\right\}_{k, p}\right]\right) e^{-\sum_{q}\left|\phi_{q}\right|^{2}} d \phi_{q} d \bar{\phi}_{q} \\
& =1+\frac{1}{Z} \int \phi_{q} \sum_{p, \tau} \operatorname{det}\left[\begin{array}{ccc}
\mid & \mid & \mid \\
B_{k \sigma, p^{\prime} \tau^{\prime}} & \frac{\partial B_{k \sigma, p \tau}}{\partial \phi_{q}} & B_{k \sigma, p^{\prime \prime} \tau^{\prime \prime}} \\
\mid & \mid & \mid
\end{array}\right] e^{-\sum_{q}\left|\phi_{q}\right|^{2}} d \phi_{q} d \bar{\phi}_{q}
\end{aligned}
$$


Since

$$
\frac{\partial}{\partial \phi_{q}} B_{k p}=\frac{i g}{\sqrt{\kappa}}\left(\begin{array}{cc}
0 & 0 \\
1 & 0
\end{array}\right) \delta_{k-p, q}
$$

we have

$$
\operatorname{det}\left[\begin{array}{ccc}
\mid & \mid & \mid \\
B_{k \sigma, p^{\prime} \tau^{\prime}} & \frac{\partial B_{k \sigma, p \tau}}{\partial \phi_{q}} & B_{k \sigma, p^{\prime \prime} \tau^{\prime \prime}} \\
\mid & \mid & \mid
\end{array}\right] / \operatorname{det}\left[\left\{B_{k p}\right\}_{k, p}\right]= \begin{cases}0 & \text { if } \tau=\downarrow \\
\frac{i g}{\sqrt{\kappa}}\left[B^{-1}\right]_{p \uparrow, p+q \downarrow} & \text { if } \tau=\uparrow\end{cases}
$$

which results in (4.22).

The inverse matrix element in $(4.22)$ we compute again with $(1.3,4)$ in the two loop approximation. Consider first the case $q=0$. Then one gets

$$
\begin{aligned}
\left\langle\left|\phi_{0}\right|^{2}\right\rangle & =1+\frac{i g}{\sqrt{\kappa}} \sum_{p} \int \phi_{0} G(p)_{\uparrow \downarrow} d P(\phi) \\
& =1+\frac{i g}{\sqrt{\kappa}} \sum_{p} \int \phi_{0} \frac{1}{\left|a_{p}+\sigma_{p}\right|^{2}+\frac{\lambda}{\kappa}\left|\phi_{0}\right|^{2}}\left(\begin{array}{cc}
\bar{a}_{p}+\bar{\sigma}_{p} & -\frac{i g}{\sqrt{\kappa}} \bar{\phi}_{0} \\
-\frac{i g}{\sqrt{\kappa}} \phi_{0} & a_{p}+\sigma_{p}
\end{array}\right)_{\uparrow \downarrow} d P(\phi) \\
& =1+\frac{\lambda}{\kappa} \sum_{p} \int \phi_{0} \frac{\bar{\phi}_{0}}{\left|a_{p}+\sigma_{p}\right|^{2}+\frac{\lambda}{\kappa}\left|\phi_{0}\right|^{2}} d P(\phi)
\end{aligned}
$$

Performing the functional integral by substitution of expectation values gives

$$
\left\langle\left|\phi_{0}\right|^{2}\right\rangle=1+\frac{\lambda}{\kappa} \sum_{p}\left\langle\left|\phi_{0}\right|^{2}\right\rangle \frac{1}{\left|a_{p}+\left\langle\sigma_{p}\right\rangle\right|^{2}+\frac{\lambda}{\kappa}\left\langle\left|\phi_{0}\right|^{2}\right\rangle}
$$

or

$$
\left\langle\left|\phi_{0}\right|^{2}\right\rangle=\frac{1}{1-\frac{\lambda}{\kappa} \sum_{p} \frac{1}{\left|a_{p}+\left\langle\sigma_{p}\right\rangle\right|^{2}+\frac{\lambda}{\kappa}\left\langle\left|\phi_{0}\right|^{2}\right\rangle}}
$$

Before we discuss (4.24), we write down the equation for $q \neq 0$. In that case we use (1.4) to compute $\left[B^{-1}(\phi)\right]_{p \uparrow, p+q \downarrow}$ in the two loop approximation. We get

$$
\begin{aligned}
& {\left[B^{-1}(\phi)\right]_{p \uparrow, p+q \downarrow} \approx-\left[G(p) B_{p, p+q} G(p+q)\right]_{\uparrow \downarrow} } \\
&=- \frac{1}{\left|a_{p}+\sigma_{p}\right|^{2}+\frac{\lambda}{\kappa}\left|\phi_{0}\right|^{2}} \frac{1}{\left|a_{p+q}+\sigma_{p+q}\right|^{2}+\frac{\lambda}{\kappa}\left|\phi_{0}\right|^{2}} \frac{i g}{\sqrt{\kappa}} \times \\
&\left(\begin{array}{cc}
-\frac{i g}{\sqrt{\kappa}}\left[(\bar{a}+\bar{\sigma})_{p+q} \bar{\phi}_{0} \phi_{-q}+(\bar{a}+\bar{\sigma})_{p} \phi_{0} \bar{\phi}_{q}\right] & (\bar{a}+\bar{\sigma})_{p}(a+\sigma)_{p+q} \bar{\phi}_{q}-\frac{\lambda}{\kappa} \bar{\phi}_{0}^{2} \phi_{-q} \\
(a+\sigma)_{p}(\bar{a}+\bar{\sigma})_{p+q} \phi_{-q}-\frac{\lambda}{\kappa} \phi_{0}^{2} \bar{\phi}_{q} & -\frac{i g}{\sqrt{\kappa}}\left[(a+\sigma)_{p+q} \phi_{0} \bar{\phi}_{q}+(a+\sigma)_{p} \bar{\phi}_{0} \phi_{-q}\right]
\end{array}\right)_{\uparrow \downarrow} \\
&=-\frac{i g}{\sqrt{\kappa}} \frac{(\bar{a}+\bar{\sigma})_{p}(a+\sigma)_{p+q} \bar{\phi}_{q}-\frac{\lambda}{\kappa} \bar{\phi}_{0}^{2} \phi_{-q}}{\left(\left|a_{p}+\sigma_{p}\right|^{2}+\frac{\lambda}{\kappa}\left|\phi_{0}\right|^{2}\right)\left(\left|a_{p+q}+\sigma_{p+q}\right|^{2}+\frac{\lambda}{\kappa}\left|\phi_{0}\right|^{2}\right)}
\end{aligned}
$$


which gives

$$
\begin{aligned}
\left\langle\left|\phi_{q}\right|^{2}\right\rangle & =1+\frac{\lambda}{\kappa} \sum_{p} \int \phi_{q} \frac{(\bar{a}+\bar{\sigma})_{p}(a+\sigma)_{p+q} \bar{\phi}_{q}-\frac{\lambda}{\kappa} \bar{\phi}_{0}^{2} \phi_{-q}}{\left(\left|a_{p}+\sigma_{p}\right|^{2}+\frac{\lambda}{\kappa}\left|\phi_{0}\right|^{2}\right)\left(\left|a_{p+q}+\sigma_{p+q}\right|^{2}+\frac{\lambda}{\kappa}\left|\phi_{0}\right|^{2}\right)} d P(\phi) \\
& =1+\frac{\lambda}{\kappa} \sum_{p} \frac{\left(\bar{a}_{p}+\left\langle\bar{\sigma}_{p}\right\rangle\right)\left(a_{p+q}+\left\langle\sigma_{p+q}\right\rangle\right)\left\langle\left|\phi_{q}\right|^{2}\right\rangle-\frac{\lambda}{\kappa}\left\langle\bar{\phi}_{0}^{2} \phi_{q} \phi_{-q}\right\rangle}{\left(\left|a_{p}+\left\langle\sigma_{p}\right\rangle\right|^{2}+\frac{\lambda}{\kappa}\left\langle\left|\phi_{0}\right|^{2}\right\rangle\right)\left(\left|a_{p+q}+\left\langle\sigma_{p+q}\right\rangle\right|^{2}+\frac{\lambda}{\kappa}\left\langle\left|\phi_{0}\right|^{2}\right\rangle\right)}
\end{aligned}
$$

Although one may think that the expectation $\left\langle\bar{\phi}_{0}^{2} \phi_{q} \phi_{-q}\right\rangle$ vanishes for zero external field, this is not so. This can be seen again by partial integration:

$$
\begin{aligned}
& \left\langle\bar{\phi}_{0}^{2} \phi_{q} \phi_{-q}\right\rangle=\frac{1}{Z} \int \bar{\phi}_{0}^{2} \phi_{q} \phi_{-q} \operatorname{det}\left[\left\{B_{k p}(\phi)\right\}_{k, p}\right] e^{-\sum_{q}\left|\phi_{q}\right|^{2}} d \phi_{q} d \bar{\phi}_{q} \\
& =\frac{1}{Z} \int \bar{\phi}_{0}^{2} \phi_{q}\left(\frac{\partial}{\partial \bar{\phi}_{-q}} \operatorname{det}\left[\left\{B_{k p}(\phi)\right\}_{k, p}\right]\right) e^{-\sum_{q}\left|\phi_{q}\right|^{2}} d \phi_{q} d \bar{\phi}_{q} \\
& =\frac{1}{Z} \int \bar{\phi}_{0}^{2} \phi_{q} \sum_{p, \tau} \operatorname{det}\left[\begin{array}{ccc}
\mid & \mid & \mid \\
B_{k \sigma, p^{\prime} \tau^{\prime}} & \frac{\partial B_{k \sigma, p \tau}}{\partial \bar{\phi}_{-q}} & B_{k \sigma, p^{\prime \prime} \tau^{\prime \prime}} \\
\mid & \mid & \mid
\end{array}\right] e^{-\sum_{q}\left|\phi_{q}\right|^{2}} d \phi_{q} d \bar{\phi}_{q}
\end{aligned}
$$

The above determinant is multiplied and devided by $\operatorname{det}\left[\left\{B_{k p}\right\}_{k, p}\right]$ to give

$$
\operatorname{det}\left[\begin{array}{ccc}
\mid & \mid & \mid \\
B_{k \sigma, p^{\prime} \tau^{\prime}} & \frac{\partial B_{k \sigma, p \tau}}{\partial \phi_{-q}} & B_{k \sigma, p^{\prime \prime} \tau^{\prime \prime}} \\
\mid & \mid & \mid
\end{array}\right] / \operatorname{det}\left[\left\{B_{k p}\right\}_{k, p}\right]= \begin{cases}0 & \text { if } \tau=\uparrow \\
\frac{i g}{\sqrt{\kappa}}\left[B^{-1}\right]_{p \downarrow, p+q \uparrow} & \text { if } \tau=\downarrow\end{cases}
$$

Computing the inverse matrix element again in the two loop approximation (4.25), we arrive at

$$
\left\langle\bar{\phi}_{0}^{2} \phi_{q} \phi_{-q}\right\rangle=\frac{\lambda}{\kappa} \sum_{p}\left\langle\frac{\left(a_{p}+\sigma_{p}\right)\left(\bar{a}_{p+q}+\sigma_{p+q}\right) \bar{\phi}_{0}^{2} \phi_{q} \phi_{-q}-\frac{\lambda}{\kappa} \bar{\phi}_{0}^{2} \phi_{0}^{2} \phi_{q} \bar{\phi}_{q}}{\left(\left|a_{p}+\sigma_{p}\right|^{2}+\frac{\lambda}{\kappa}\left|\phi_{0}\right|^{2}\right)\left(\left|a_{p+q}+\sigma_{p+q}\right|^{2}+\frac{\lambda}{\kappa}\left|\phi_{0}\right|^{2}\right)}\right\rangle
$$

Abbreviating

$$
g_{p}=\frac{a_{p}+\left\langle\sigma_{p}\right\rangle}{\left|a_{p}+\left\langle\sigma_{p}\right\rangle\right|^{2}+\frac{\lambda}{\kappa}\left\langle\left|\phi_{0}\right|^{2}\right\rangle}, \quad f_{p}=\frac{\sqrt{\frac{\lambda}{\kappa}\left\langle\left|\phi_{0}\right|^{2}\right\rangle}}{\left|a_{p}+\left\langle\sigma_{p}\right\rangle\right|^{2}+\frac{\lambda}{\kappa}\left\langle\left|\phi_{0}\right|^{2}\right\rangle}
$$

this gives

$$
\frac{\lambda}{\kappa}\left\langle\bar{\phi}_{0}^{2} \phi_{q} \phi_{-q}\right\rangle=\frac{\lambda}{\kappa} \sum_{p} g_{p} \bar{g}_{p+q} \frac{\lambda}{\kappa}\left\langle\bar{\phi}_{0}^{2} \phi_{q} \phi_{-q}\right\rangle-\frac{\lambda}{\kappa} \sum_{p} f_{p} f_{p+q} \frac{\lambda}{\kappa}\left\langle\left|\phi_{0}\right|^{2}\right\rangle\left\langle\left|\phi_{q}\right|^{2}\right\rangle
$$

or

$$
\frac{\lambda}{\kappa}\left\langle\bar{\phi}_{0}^{2} \phi_{q} \phi_{-q}\right\rangle=\frac{-\frac{\lambda}{\kappa} \sum_{p} f_{p} f_{p+q} \frac{\lambda}{\kappa}\left\langle\left|\phi_{0}\right|^{2}\right\rangle}{1-\frac{\lambda}{\kappa} \sum_{p} g_{p} \bar{g}_{p+q}}\left\langle\left|\phi_{q}\right|^{2}\right\rangle
$$

Substituting this in (4.26), we finally arrive at

$$
\left\langle\left|\phi_{q}\right|^{2}\right\rangle=\frac{1-\frac{\lambda}{\kappa} \sum_{p} g_{p} \bar{g}_{p+q}}{\left|1-\frac{\lambda}{\kappa} \sum_{p} g_{p} \bar{g}_{p+q}\right|^{2}-\left(\frac{\lambda}{\kappa} \sum_{p} f_{p} f_{p+q}\right)^{2}}
$$


where $g_{p}, f_{p}$ are given by (4.27). Observe that, since $d P(\phi)$ is complex, also $\left\langle\left|\phi_{q}\right|^{2}\right\rangle$ is in general complex. Only after summation over the $q_{0}$ variables we obtain necessarily a real quantity which is given by $(4.4,11)$.

We now discuss the solutions to (4.24) and (4.29). We assume that the solution $\left\langle\sigma_{k}\right\rangle$ of (4.21) is sufficiently small such that the BCS equation

$$
\frac{\lambda}{\kappa} \sum_{p} \frac{1}{\left|a_{p}+\left\langle\sigma_{p}\right\rangle\right|^{2}+|\Delta|^{2}}=1
$$

has a nonzero solution $\Delta \neq 0$ (in particular this excludes large corrections like $\left\langle\sigma_{p}\right\rangle \sim p_{0}^{\alpha}$, $\alpha \leq 1 / 2$, which one may expect in the case of Luttinger liquid behaviour, for $d=1$ one should make a seperate analysis), and make the Ansatz

$$
\lambda\left\langle\left|\phi_{0}\right|^{2}\right\rangle=\beta L^{d}|\Delta|^{2}+\eta
$$

where $\eta$ is independent of the volume. Then

$$
\begin{aligned}
\frac{\lambda}{\kappa} \sum_{p} \frac{1}{\left|a_{p}+\left\langle\sigma_{p}\right\rangle\right|^{2}+\frac{\lambda}{\kappa}\left\langle\left|\phi_{0}\right|^{2}\right\rangle} & =\frac{\lambda}{\kappa} \sum_{p} \frac{1}{\left|a_{p}+\left\langle\sigma_{p}\right\rangle\right|^{2}+|\Delta|^{2}+\frac{\eta}{\kappa}} \\
& =\frac{\lambda}{\kappa} \sum_{p} \frac{1}{\left|a_{p}+\left\langle\sigma_{p}\right\rangle\right|^{2}+|\Delta|^{2}}-\frac{\lambda}{\kappa} \sum_{p} \frac{\eta / \kappa}{\left(\left|a_{p}+\left\langle\sigma_{p}\right\rangle\right|^{2}+|\Delta|^{2}\right)^{2}}+O\left(\left(\frac{\eta}{\kappa}\right)^{2}\right) \\
& =1-c_{\Delta} \frac{\eta}{\kappa}+O\left(\left(\frac{\eta}{\kappa}\right)^{2}\right)
\end{aligned}
$$

where we put $c_{\Delta}=\frac{\lambda}{\kappa} \sum_{p} \frac{1}{\left(\left|a_{p}+\left\langle\sigma_{p}\right\rangle\right|^{2}+|\Delta|^{2}\right)^{2}}$ and used the BCS equation (4.30) in the last line. Equation (4.24) becomes

$$
\kappa|\Delta|^{2}+\eta=\frac{\lambda}{c_{\Delta} \frac{\eta}{\kappa}+O\left(\left(\frac{\eta}{\kappa}\right)^{2}\right)}=\kappa \frac{\lambda}{c_{\Delta} \eta}+O(1)
$$

and has a solution $\eta=\lambda /\left(c_{\Delta}|\Delta|^{2}\right)$.

Now consider $\left\langle\left|\phi_{q}\right|^{2}\right\rangle$ for small but nonzero $q$. In the limit $q \rightarrow 0$ the denominator in (4.29) vanishes, or more precisely, is of order $O(1 / \kappa)$ since

$$
1-\frac{\lambda}{\kappa} \sum_{p} g_{p} \bar{g}_{p}-\frac{\lambda}{\kappa} \sum_{p} f_{p} f_{p}=1-\frac{\lambda}{\kappa} \sum_{p} \frac{1}{\left|a_{p}+\left\langle\sigma_{p}\right\rangle\right|^{2}+\frac{\lambda}{\kappa}\left\langle\left|\phi_{0}\right|^{2}\right\rangle}=O(1 / \kappa)
$$

because of (4.32). If we assume the second derivatives of $\left\langle\sigma_{k}\right\rangle$ to be integrable (which should be the case for $d=3$ and $\left\langle\left|\phi_{q}\right|^{2}\right\rangle \sim 1 / q^{2}$ by virtue of (4.21)), then, since the denominator in (4.29) is an even function of $q$, the small $q$ behaviour of $\left\langle\left|\phi_{q}\right|^{2}\right\rangle$ is $1 / q^{2}$. This agrees with the common expectations [FMRT,CFS,B]. Usually the behaviour of $\left\langle\left|\phi_{q}\right|^{2}\right\rangle$ is infered from the second order Taylor expansion of the effective potential

$$
V_{\text {eff }}\left(\left\{\phi_{q}\right\}\right)=\sum_{q}\left|\phi_{q}\right|^{2}-\log \operatorname{det}\left[\begin{array}{cc}
\delta_{k, p} & \frac{i g}{\sqrt{\kappa}} \frac{\bar{\phi}_{p-k}}{a_{k}} \\
\frac{i g}{\sqrt{\kappa}} \frac{\phi_{k-p}}{a_{-k}} & \delta_{k, p}
\end{array}\right]
$$


around its global minimum [L2]

$$
\phi_{q}^{\min }=\sqrt{\beta L^{d}} \frac{|\Delta|}{\sqrt{\lambda}} \delta_{q, 0} e^{i \theta_{0}}
$$

where the phase $\theta_{0}$ of $\phi_{0}$ is arbitrary. If one expands $V_{\text {eff }}$ up to second order in

$$
\xi_{q}=\phi_{q}-\delta_{q, 0} \sqrt{\beta L^{d}} \frac{|\Delta|}{\sqrt{\lambda}} e^{i \theta_{0}}= \begin{cases}\left(\rho_{0}-\sqrt{\beta L^{d}} \frac{|\Delta|}{\sqrt{\lambda}}\right) e^{i \theta_{0}} & \text { for } q=0 \\ \rho_{q} e^{i \theta_{q}} & \text { for } q \neq 0\end{cases}
$$

one obtains [L2]

$$
\begin{aligned}
V_{\mathrm{eff}}\left(\left\{\phi_{q}\right\}\right)= & V_{\min }+2 \beta_{0}\left(\rho_{0}-\sqrt{\beta L^{d}} \frac{|\Delta|}{\sqrt{\lambda}}\right)^{2}+\sum_{q \neq 0}\left(\alpha_{q}+i \gamma_{q}\right) \rho_{q}^{2} \\
& +\frac{1}{2} \sum_{q \neq 0} \beta_{q}\left|e^{-i \theta_{0}} \phi_{q}+e^{i \theta_{0}} \bar{\phi}_{-q}\right|^{2}+O\left(\xi^{3}\right)
\end{aligned}
$$

where for small $q$ one has $\alpha_{q}, \gamma_{q} \sim q^{2}$. Hence, if $V_{\text {eff }}$ is substituted by the right hand side of (4.36) one obtains $\left\langle\left|\phi_{q}\right|^{2}\right\rangle \sim 1 / q^{2}$.

For $d=3$, this seems to be the right answer, but in lower dimensions one would expect an integrable singularity due to $(4.21)$ and $(4.3,4,11)$. In particular, we think it would be a very interesting problem to solve the integral equations $(4.21,24,29)$ for $d=1$ and to check the result for Luttinger liquid behaviour. A good warm up excercise would be to consider the $0+1$ dimensional problem, that is, we only have the $k_{0}, p_{0}, q_{0}$-variables. In that case the 'bare BCS equation'

$$
\frac{\lambda}{\beta} \sum_{p_{0} \in \frac{\pi}{\beta}(2 \mathbb{Z}+1)} \frac{1}{p_{0}^{2}+|\Delta|^{2}}=1
$$

still has a nonzero solution $\Delta$ for sufficiently small $T=1 / \beta$ and the question would be whether the correction $\left\langle\sigma_{p_{0}}\right\rangle$ is sufficiently big to destroy the gap. That is, does the 'renormalized BCS equation'

$$
\frac{\lambda}{\beta} \sum_{p_{0} \in \frac{\pi}{\beta}(2 \mathbb{Z}+1)} \frac{1}{\left|p_{0}+\left\langle\sigma_{p_{0}}\right\rangle\right|^{2}+|\Delta|^{2}}=1
$$

$\left\langle\sigma_{p_{0}}\right\rangle$ being the solution to $(4.21,24,29)$, still have a nonzero solution? We remark that, if the gap vanishes (for arbitrary dimension), then also the singularity of $\left\langle\left|\phi_{q}\right|^{2}\right\rangle$ disappears. Namely, if the gap equation has no solution, that is, if $\frac{1}{\kappa} \sum_{p} \frac{1}{\left|a_{p}+\left\langle\sigma_{p}\right\rangle\right|^{2}}<\infty$, then $\left\langle\left|\phi_{0}\right|^{2}\right\rangle$ given by (4.24) is no longer macroscopic (for sufficiently small coupling) and $\frac{\lambda}{\kappa}\left\langle\left|\phi_{0}\right|^{2}\right\rangle$ vanishes in the infinite volume limit. And the denominator in (4.29) becomes for $q \rightarrow 0$

$$
1-\frac{\lambda}{\kappa} \sum_{p} \frac{1}{\left|a_{p}+\left\langle\sigma_{p}\right\rangle\right|^{2}}
$$


which would be nonzero (for sufficiently small coupling).

Finally we argue why it is reasonable to substitute $\left|\phi_{0}\right|^{2}$ by its expectation value while performing the functional integral. We may write the effective potential (4.33) as

$$
V_{\mathrm{eff}}\left(\left\{\phi_{q}\right\}\right)=V_{1}\left(\phi_{0}\right)+V_{2}\left(\left\{\phi_{q}\right\}\right)
$$

where

$$
\begin{aligned}
V_{1}\left(\phi_{0}\right) & =\left|\phi_{0}\right|^{2}-\sum_{k} \log \left[1+\frac{\lambda}{\kappa} \frac{\left|\phi_{0}\right|^{2}}{k_{0}^{2}+e_{\mathbf{k}}^{2}}\right] \\
& =\kappa\left(\frac{\left|\phi_{0}\right|^{2}}{\kappa}-\frac{1}{\kappa} \sum_{k} \log \left[1+\frac{\lambda \frac{\left|\phi_{0}\right|^{2}}{\kappa}}{k_{0}^{2}+e_{\mathbf{k}}^{2}}\right]\right) \equiv \kappa V_{\mathrm{BCS}}\left(\frac{\left|\phi_{0}\right|}{\sqrt{\kappa}}\right)
\end{aligned}
$$

and

$$
V_{2}\left(\left\{\phi_{q}\right\}\right)=\sum_{q \neq 0}\left|\phi_{q}\right|^{2}-\log \operatorname{det}\left[\left(\begin{array}{cc}
\delta_{k, p} & \frac{i g}{\sqrt{\kappa}} \frac{\bar{\phi}_{0}}{a_{k}} \delta_{k, p} \\
\frac{i g}{\sqrt{\kappa}} \frac{\phi_{0}}{a_{-k}} \delta_{k, p} & \delta_{k, p}
\end{array}\right)^{-1}\left(\begin{array}{cc}
\delta_{k, p} & \frac{i g}{\sqrt{\kappa}} \frac{\bar{\phi}_{p-k}}{a_{k}} \\
\frac{i g}{\sqrt{\kappa}} \frac{\phi_{k-p}}{a_{-k}} & \delta_{k, p}
\end{array}\right)\right]
$$

If we ignore the $\phi_{0}$-dependence of $V_{2}$, then the $\phi_{0}$-integral

$$
\frac{\int F\left(\frac{1}{\kappa}\left|\phi_{0}\right|^{2}\right) e^{-V_{1}\left(\phi_{0}\right)} d \phi_{0} d \bar{\phi}_{0}}{\int e^{-V_{1}\left(\phi_{0}\right)} d \phi_{0} d \bar{\phi}_{0}}=\frac{\int F\left(\rho^{2}\right) e^{-\kappa V_{\mathrm{BCS}}(\rho)} \rho d \rho}{\int e^{-\kappa V_{\mathrm{BCS}}(\rho)} \rho d \rho} \stackrel{\kappa \rightarrow \infty}{\rightarrow} F\left(\rho_{\min }^{2}\right)=F\left(\frac{1}{\kappa}\left\langle\left|\phi_{0}\right|^{2}\right\rangle\right)
$$

simply puts $\left|\phi_{0}\right|^{2}$ at the global minimum of the (BCS) effective potential.

\subsection{The $\varphi^{4}$-Model}

In this section we choose the $\varphi^{4}$-model as a typical bosonic model to demonstrate our method. As in section 2, we start in finite volume $[0, L]^{d}$ on a lattice with lattice spacing $1 / M$. The two point function is given by

$$
S(x, y)=\left\langle\varphi_{x} \varphi_{y}\right\rangle:=\frac{\int_{\mathbb{R}^{N^{d}}} \varphi_{x} \varphi_{y} e^{-\frac{g^{2}}{2} \frac{1}{M^{d}} \sum_{x} \varphi_{x}^{4}} e^{-\frac{1}{M^{2 d}} \sum_{x, y}\left(-\Delta+m^{2}\right)_{x, y} \varphi_{x} \varphi_{y}} \Pi_{x} d \varphi_{x}}{\int_{\mathbb{R}^{N^{d}}} e^{-\frac{g^{2}}{2} \frac{1}{M^{d}} \sum_{x} \varphi_{x}^{4}} e^{-\frac{1}{M^{2 d}} \sum_{x, y}\left(-\Delta+m^{2}\right)_{x, y} \varphi_{x} \varphi_{y}} \Pi_{x} d \varphi_{x}}
$$

where

$$
\left(-\Delta+m^{2}\right)_{x, y}=M^{d}\left[-M^{2} \sum_{i=1}^{d}\left(\delta_{x, y-e_{i} / M}+\delta_{x, y+e_{i} / M}-2 \delta_{x, y}\right)+m^{2} \delta_{x, y}\right]
$$


First we have to bring this into the form $\int[P+Q]_{x, y}^{-1} d \mu, P$ diagonal in momentum space, $Q$ diagonal in coordinate space. This is done again by making a Hubbard Stratonovich transformation which in this case reads

$$
e^{-\frac{1}{2} \sum_{x} a_{x}^{2}}=\int e^{i \sum_{x} a_{x} u_{x}} e^{-\frac{1}{2} \sum_{x} u_{x}^{2}} \prod_{x} \frac{d u_{x}}{\sqrt{2 \pi}}
$$

with

$$
a_{x}=\frac{g}{\sqrt{M^{d}}} \varphi_{x}^{2}
$$

The result is Gaussian in the $\varphi_{x}$-variables and the integral over these variables gives

$$
S(x, y)=\int_{\mathbb{R}^{N^{d}}}\left[\frac{1}{M^{2 d}}\left(-\Delta+m^{2}\right)_{x, y}-\frac{i g}{\sqrt{M^{d}}} u_{x} \delta_{x, y}\right]_{x, y}^{-1} d P(u)
$$

where

$$
d P(u)=\frac{1}{Z} \operatorname{det}\left[\frac{1}{M^{2 d}}\left(-\Delta+m^{2}\right)_{x, y}-\frac{i g}{\sqrt{M^{d}}} u_{x} \delta_{x, y}\right]^{-\frac{1}{2}} e^{-\frac{1}{2} \sum_{x} u_{x}^{2}} \prod_{x} d u_{x}
$$

Since we have bosons, the determinant comes with a power of $-1 / 2$ which is the only difference compared to a fermionic system. In momentum space this reads (compare equations (2.7-11))

$$
S(x-y)=\frac{1}{L^{d}} \sum_{k} e^{i k(x-y)}\langle G\rangle(k)
$$

where $\left(\gamma_{q}=v_{q}+i w_{q}, \gamma_{-q}=\bar{\gamma}_{q}, d \gamma_{q} d \bar{\gamma}_{q}:=d v_{q} d w_{q}\right)$

$$
\langle G\rangle(k)=\int_{\mathbb{R}^{N^{d}}}\left[a_{k} \delta_{k, p}-\frac{i g}{\sqrt{L^{d}}} \gamma_{k-p}\right]_{k k}^{-1} d P(\gamma)
$$

and

$$
d P(\gamma)=\frac{1}{Z} \operatorname{det}\left[a_{k} \delta_{k, p}-\frac{i g}{\sqrt{L^{d}}} \gamma_{k-p}\right]^{-\frac{1}{2}} e^{-\frac{1}{2} v_{0}^{2}} d v_{0} \prod_{q \in \mathcal{M}^{+}} e^{-\left|\gamma_{q}\right|^{2}} d \gamma_{q} d \bar{\gamma}_{q}
$$

and $\mathcal{M}^{+}$again is a set such that either $q \in \mathcal{M}^{+}$or $-q \in \mathcal{M}^{+}$. Furthermore

$$
a_{k}=4 M^{2} \sum_{i=1}^{d} \sin ^{2}\left[\frac{k_{i}}{2 M}\right]+m^{2}
$$

Equation (4.48) is our starting point. We apply (1.3) to the inverse matrix element in (4.48). In the two loop approximation one obtains $\left(\gamma_{0}=v_{0} \in \mathbb{R}\right)$

$$
\left[a_{k} \delta_{k, p}-\frac{i g}{\sqrt{L^{d}}} \gamma_{k-p}\right]_{k k}^{-1} \approx \frac{1}{a_{k}-\frac{i g v_{0}}{\sqrt{L^{d}}}+\frac{g^{2}}{L^{d}} \sum_{p \neq k} G_{k}(p)\left|\gamma_{k-p}\right|^{2}}=: \frac{1}{a_{k}+\sigma_{k}}
$$

where

$$
\sigma_{k}=-\frac{i g}{\sqrt{L^{d}}} v_{0}+\frac{g^{2}}{L^{d}} \sum_{p \neq k} \frac{\left|\gamma_{k-p}\right|^{2}}{a_{p}-\frac{i g v_{0}}{\sqrt{L^{d}}}+\sigma_{p}}
$$


which results in

$$
\langle G\rangle(k)=\frac{1}{a_{k}+\left\langle\sigma_{k}\right\rangle}
$$

where $\left\langle\sigma_{k}\right\rangle$ has to satisfy the equation

$$
\begin{aligned}
\left\langle\sigma_{k}\right\rangle & =-\frac{i g}{\sqrt{L^{d}}}\left\langle v_{0}\right\rangle+\frac{g^{2}}{L^{d}} \sum_{p \neq k} \frac{\left\langle\left|\gamma_{k-p}\right|^{2}\right\rangle}{a_{p}+\left\langle\sigma_{p}\right\rangle} \\
& =\frac{g^{2}}{2 L^{d}} \sum_{p}\langle G\rangle(p)+\frac{g^{2}}{L^{d}} \sum_{p \neq k} \frac{\left\langle\left|\gamma_{k-p}\right|^{2}\right\rangle}{a_{p}+\left\langle\sigma_{p}\right\rangle}=\frac{g^{2}}{L^{d}} \sum_{p \neq k} \frac{\left\langle\left|\gamma_{k-p}\right|^{2}\right\rangle+\frac{1}{2}}{a_{p}+\left\langle\sigma_{p}\right\rangle}
\end{aligned}
$$

where the last line is due to

$$
\begin{aligned}
& \left\langle v_{0}\right\rangle=\frac{1}{Z} \int v_{0} \operatorname{det}\left[a_{k} \delta_{k, p}-\frac{i g}{\sqrt{L^{d}}} \gamma_{k-p}\right]^{-\frac{1}{2}} e^{-\frac{1}{2} v_{0}^{2}} d v_{0} \prod_{q \in \mathcal{M}^{+}} e^{-\left|\gamma_{q}\right|^{2}} d \gamma_{q} d \bar{\gamma}_{q} \\
& =\frac{1}{Z} \int\left\{\frac{\partial}{\partial v_{0}} \operatorname{det}\left[a_{k} \delta_{k, p}-\frac{i g}{\sqrt{L^{d}}} \gamma_{k-p}\right]^{-\frac{1}{2}}\right\} e^{-\frac{1}{2} v_{0}^{2}} d v_{0} \prod_{q \in \mathcal{M}^{+}} e^{-\left|\gamma_{q}\right|^{2}} d \gamma_{q} d \bar{\gamma}_{q} \\
& =-\frac{1}{2} \sum_{p}\left(-\frac{i g}{\sqrt{L^{d}}}\right) \int\left[a_{k} \delta_{k, p}-\frac{i g}{\sqrt{L^{d}}} \gamma_{k-p}\right]_{p p}^{-1} d P(\gamma)
\end{aligned}
$$

As for the Many-Electron system, we can derive an equation for $\left\langle\left|\gamma_{q}\right|^{2}\right\rangle$ by partial integration:

$$
\begin{aligned}
\left\langle\left|\gamma_{q}\right|^{2}\right\rangle & =\frac{1}{Z} \int \gamma_{q} \bar{\gamma}_{q} \operatorname{det}\left[a_{k} \delta_{k, p}-\frac{i g}{\sqrt{L^{d}}} \gamma_{k-p}\right]^{-\frac{1}{2}} e^{-\frac{v_{0}^{2}}{2}} d v_{0} \prod_{q} e^{-\left|\gamma_{q}\right|^{2}} d \gamma_{q} d \bar{\gamma}_{q} \\
& =1+\frac{1}{Z} \int \gamma_{q} \frac{\partial}{\partial \gamma_{q}}\left\{\operatorname{det}\left[a_{k} \delta_{k, p}-\frac{i g}{\sqrt{L^{d}}} \gamma_{k-p}\right]^{-\frac{1}{2}}\right\} e^{-\frac{v_{0}^{2}}{2}} d v_{0} \prod_{q} e^{-\frac{1}{2}\left|\gamma_{q}\right|^{2}} d \gamma_{q} d \bar{\gamma}_{q} \\
& =1-\frac{1}{2} \int \gamma_{q} \frac{\frac{\partial}{\partial \gamma_{q}} \operatorname{det}\left[a_{k} \delta_{k, p}-\frac{i g}{\sqrt{L^{d}}} \gamma_{k-p}\right]}{\operatorname{det}\left[a_{k} \delta_{k, p}-\frac{i g}{\sqrt{L^{d}}} \gamma_{k-p}\right]} d P(\gamma) \\
& =1-\frac{1}{2} \sum_{p} \frac{-i g}{\sqrt{L^{d}}} \int \gamma_{q}\left[a_{k} \delta_{k, p}-\frac{i g}{\sqrt{L^{d}}} \gamma_{k-p}\right]_{p, p+q}^{-1} d P(\gamma)
\end{aligned}
$$

Computing the inverse matrix element in (4.56) again in the two loop approximation, one arrives at

$$
\left\langle\left|\gamma_{q}\right|^{2}\right\rangle=1-\left\langle\left|\gamma_{q}\right|^{2}\right\rangle \frac{g^{2}}{2 L^{d}} \sum_{p} \frac{1}{\left(a_{p}+\left\langle\sigma_{p}\right\rangle\right)\left(a_{p+q}+\left\langle\sigma_{p+q}\right\rangle\right)}
$$

or

$$
\left\langle\left|\gamma_{q}\right|^{2}\right\rangle=\frac{1}{1+\frac{g^{2}}{2} \int_{[0,2 \pi M]^{d}} \frac{d^{d} p}{(2 \pi)^{d}} \frac{1}{\left(a_{p}+\left\langle\sigma_{p}\right\rangle\right)\left(a_{p+q}+\left\langle\sigma_{p+q}\right\rangle\right)}}
$$

which has to be solved in conjunction with

$$
\left\langle\sigma_{k}\right\rangle=g^{2} \int_{[0,2 \pi M]^{d}} \frac{d^{d} p}{(2 \pi)^{d}} \frac{\left\langle\left|\gamma_{k-p}\right|^{2}\right\rangle+\frac{1}{2}}{a_{p}+\left\langle\sigma_{p}\right\rangle}
$$


Introducing the rescaled quantities

$$
\left\langle\sigma_{k}\right\rangle=M^{2} s_{\frac{p}{M}}, \quad\left\langle\left|\gamma_{q}\right|^{2}\right\rangle=\lambda_{\frac{q}{M}}, \quad a_{k}=M^{2} \varepsilon_{\frac{k}{M}}, \quad \varepsilon_{k}=\sum_{i=1}^{d} \sin ^{2} \frac{k_{i}}{2}+\frac{m^{2}}{M^{2}}
$$

$(4.57,58) \mathrm{read}$

$$
\begin{aligned}
& s_{k}=M^{d-4} g^{2} \int_{[0,2 \pi]^{d}} \frac{d^{d} p}{(2 \pi)^{d}} \frac{\lambda_{k-p}+\frac{1}{2}}{\varepsilon_{p}+s_{p}} \\
& \lambda_{q}=\frac{1}{1+M^{d-4} \frac{g^{2}}{2} \int_{[0,2 \pi]^{d}} \frac{d^{d} p}{(2 \pi)^{d}} \frac{1}{\left(\varepsilon_{p}+s_{p}\right)\left(\varepsilon_{p+q}+s_{p+q}\right)}}
\end{aligned}
$$

Unfortunately we cannot check this result with the rigorously proven triviality theorem since $\left\langle\sigma_{k}\right\rangle$ and $\left\langle\left|\gamma_{q}\right|^{2}\right\rangle$ only give information on the 2-point function $S(x, y)$, (4.41), and on $\frac{g^{2}}{M^{d}} \sum_{x}\left\langle\varphi(x)^{4}\right\rangle=\sum_{q} \Lambda(q)$ where $\Lambda(q)=\left\langle\left|\gamma_{q}\right|^{2}\right\rangle-1$. However, the triviality theorem $[\mathrm{F}, \mathrm{FFS}]$ makes a statement on the connected 4-point function $S_{4, c}\left(x_{1}, x_{2}, x_{3}, x_{4}\right)$ at noncoinciding arguments, namely that this function vanishes in the continuum limit in dimension $d>4$.

Before we include the higher loop terms of $(1.3,4)$ and give an interpretation in terms of diagrams, we would like to comment shortly on a problem which was suggested to us by A. Sokal after a preprint of this paper was published on the web. It refers to the $\phi^{2} \psi^{2}$ or $\phi_{1}^{2} \phi_{2}^{2}$-model. That is, we have two scalar bosonic fields on a lattice with unit lattice spacing with action

$$
\mathcal{S}\left(\phi_{1}, \phi_{2}\right)=\sum_{i=1}^{2} \sum_{x} \phi_{i}(x)\left(-\Delta+m^{2}\right) \phi_{i}(x)+\lambda \sum_{x} \phi_{1}(x)^{2} \phi_{2}(x)^{2}
$$

The question is whether there is exponential decay (or a gap in momentum space) for the two point function $G(x, y)=\int \phi_{1}(x) \phi_{1}(y) e^{-\mathcal{S}(\phi)} / \int e^{-\mathcal{S}(\phi)}$ in the zero mass $m \rightarrow 0$ limit. A computation with the above formalism in two loop approximation gives $G(k)=\frac{1}{k^{2}+\sigma}$ where the gap $\sigma$ has to satisfy the equation $\sigma=\lambda \int_{[-\pi, \pi]^{d}} \frac{d^{d} p}{(2 \pi)^{d}} \frac{1}{p^{2}+\sigma}$ which gives

$$
\sigma=\left\{\begin{aligned}
O(\lambda) & \text { if } d \geq 3 \\
O(\lambda \log [1 / \lambda]) & \text { if } d=2 \\
O\left(\lambda^{\frac{2}{3}}\right) & \text { if } d=1
\end{aligned}\right.
$$

We now include the higher loop terms of $(1.3,4)$ and give an interpretation in terms of diagrams. The exact equations for $\langle G\rangle(k)$ and $\left\langle\left|\gamma_{q}\right|^{2}\right\rangle$ are

$$
\begin{aligned}
\langle G\rangle(k) & =\int\left[a_{k} \delta_{k, p}-\frac{i g}{\sqrt{L^{d}}} \gamma_{k-p}\right]_{k k}^{-1} d P(\gamma)=\left\langle\frac{1}{a_{k}+\sigma_{k}}\right\rangle \\
\sigma_{k} & =-\frac{i g}{\sqrt{L^{d}}} v_{0}+\sum_{r=2}^{N^{d}}\left(\frac{i g}{\sqrt{L^{d}}}\right)_{\substack{p_{2} \cdots p_{r} \neq k \\
p_{i} \neq p_{j}}}^{r} G_{k}\left(p_{2}\right) \cdots G_{k p_{2} \cdots p_{r-1}}\left(p_{r}\right) \gamma_{k-p_{2}} \gamma_{p_{2}-p_{3}} \cdots \gamma_{p_{r}-k}
\end{aligned}
$$


and

$$
\begin{aligned}
&\left\langle\left|\gamma_{q}\right|^{2}\right\rangle=1+\frac{i g}{2 \sqrt{L^{d}}} \sum_{p} \int \gamma_{q}\left[a_{k} \delta_{k, p}-\frac{i g}{\sqrt{L^{d}}} \gamma_{k-p}\right]_{p, p+q}^{-1} d P(\gamma) \\
& \stackrel{p \rightarrow p_{2}}{=} 1+\frac{1}{2} \sum_{r=2}^{N^{d}}\left(\frac{i g}{\sqrt{L^{d}}}\right)_{\substack{p_{2} \cdots p_{r \neq p} \neq p_{2}+q \\
p_{i} \neq p_{j}}}^{r}\left\langle G\left(p_{2}\right) G_{p_{2}}\left(p_{3}\right) \cdots G_{p_{2} \cdots p_{r-1}}\left(p_{r}\right) G_{p_{2} \cdots p_{r}}\left(p_{2}+q\right) \times\right. \\
&\left.\gamma_{p_{2}-p_{3}} \cdots \gamma_{p_{r-1}-p_{r}} \gamma_{p_{r}-p_{2}-q} \gamma_{p_{2}+q-p_{2}}\right\rangle
\end{aligned}
$$

For $r>2$, we obtain terms $\left\langle\gamma_{k_{1}} \cdots \gamma_{k_{r}}\right\rangle$ whose connected contributions
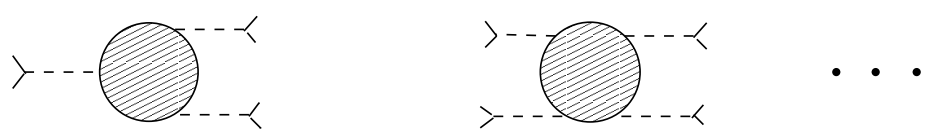

Figure 4: three and higher loop contributions

are, in terms of the electron or $\varphi^{4}$-lines, are at least six-legged. Since for the manyelectron system and for the $\varphi^{4}$-model (for $d=4$ ) the relevant diagrams are two- and four-legged $[\mathrm{FT}, \mathrm{R}]$, one may start with an approximation which ignores the connected $r$-loop contributions for $r>2$. This is obtained by writing

$$
\left\langle\gamma_{k_{1}} \cdots \gamma_{k_{n}}\right\rangle \approx\left\langle\gamma_{k_{1}} \cdots \gamma_{k_{n}}\right\rangle_{2}
$$

where (the index '2' for 'retaining only two-loop contributions')

$$
\left\langle\gamma_{k_{1}} \cdots \gamma_{k_{2 n}}\right\rangle_{2}:=\sum_{\text {pairings } \sigma}\left\langle\gamma_{k_{\sigma 1}} \gamma_{k_{\sigma 2}}\right\rangle \cdots\left\langle\gamma_{k_{\sigma(2 n-1)}} \gamma_{k_{\sigma 2 n}}\right\rangle=\int \gamma_{k_{1}} \cdots \gamma_{k_{2 n}} d P_{2}(\gamma)
$$

if we define

$$
d P_{2}(\gamma):=e^{-\sum_{q} \frac{\left|\gamma_{q}\right|^{2}}{\left\langle\left\langle\left.\gamma_{q}\right|^{2}\right\rangle\right.}} \prod_{q} \frac{d \gamma_{q} d \bar{\gamma}_{q}}{\pi\left\langle\left|\gamma_{q}\right|^{2}\right\rangle}
$$

Substituting $d P$ by $d P_{2}$ in $(4.63,64)$, we obtain a model which differs from the original model only by irrelevant contributions and for which we are able to write down a closed set of equations for the two-legged particle correlation function $\langle G\rangle(k)$ and the two-legged squiggle correlation function $\left\langle\left|\gamma_{q}\right|^{2}\right\rangle$ by resumming all two-legged (particle and squiggle) subdiagrams. The exact equations of this model are

$$
\begin{aligned}
\langle G\rangle(k) & =\int\left[a_{k} \delta_{k, p}-\frac{i g}{\sqrt{L^{d}}} \gamma_{k-p}\right]_{k k}^{-1} d P_{2}(\gamma) \\
\left\langle\left|\gamma_{q}\right|^{2}\right\rangle & =1+\frac{i g}{2 \sqrt{L^{d}}} \sum_{p} \int \gamma_{q}\left[a_{k} \delta_{k, p}-\frac{i g}{\sqrt{L^{d}}} \gamma_{k-p}\right]_{p, p+q}^{-1} d P_{2}(\gamma)
\end{aligned}
$$


and the resummation of the two-legged particle and squiggle subdiagrams is obtained by applying the inversion formula $(1.3,4)$ to the inverse matrix elements in $(4.68,69)$. A discussion similar to those of section 2 gives the following closed set of equations for the quantities $\langle G\rangle(k)$ and $\left\langle\left|\gamma_{q}\right|^{2}\right\rangle$ :

$$
\langle G\rangle(k)=\frac{1}{a_{k}+\left\langle\sigma_{k}\right\rangle}, \quad\left\langle\left|\gamma_{q}\right|^{2}\right\rangle=\frac{1}{1+\left\langle\pi_{q}\right\rangle}
$$

where

$$
\begin{aligned}
\left\langle\sigma_{k}\right\rangle=\frac{g^{2}}{2 L^{d}} \sum_{p}\langle G\rangle(p)+\sum_{r=2}^{\ell}\left(\frac{i g}{\sqrt{L^{d}}}\right)^{r} \sum_{\substack{p_{2} \cdots p_{r} \neq k \\
p_{i} \neq p_{j}}}\langle G\rangle\left(p_{2}\right) \cdots\langle G\rangle\left(p_{r}\right)\left\langle\gamma_{k-p_{2}} \gamma_{p_{2}-p_{3}} \cdots \gamma_{p_{r}-k}\right\rangle_{2} \\
\left\langle\pi_{q}\right\rangle=-\frac{1}{2} \sum_{r=2}^{\ell}\left(\frac{i g}{\sqrt{L^{d}}}\right)^{r} \sum_{s=3}^{r-1} \sum_{\substack{p_{2} \cdots p_{r} \neq p_{2}+q \\
p_{i} \neq p_{j}}}\left(\delta_{q, p_{s+1}-p_{s}}\langle G\rangle\left(p_{2}\right) \cdots\langle G\rangle\left(p_{r}\right)\langle G\rangle\left(p_{2}+q\right) \times\right. \\
\left.\left\langle\gamma_{p_{2}-p_{3}} \cdots \widehat{\gamma}_{p_{s}-p_{s+1}} \cdots \gamma_{p_{r-1}-p_{r}} \gamma_{p_{r}-p_{2}-q}\right\rangle_{2}\right)
\end{aligned}
$$

In the last line we used that $\gamma_{q}$ in (4.64) cannot contract to $\gamma_{p_{2}-p_{3}}$ or to $\gamma_{p_{r}-p_{2}-q}$. If the expectations of the $\gamma$-fields on the right hand side of $(4.71,72)$ are computed according to (4.66), one obtains the expansion into diagrams. The graphs contributing to $\left\langle\sigma_{k}\right\rangle$ have exactly one string of particle lines, each line having $\langle G\rangle$ as propagator, and no particle loops (up to the tadpole diagram). Each squiggle corresponds to a factor $\left\langle|\gamma|^{2}\right\rangle$. The diagrams contributing to $\langle\pi\rangle$ have exactly one particle loop, the propagators being again the interacting two point functions, $\langle G\rangle$ for the particle lines and $\left\langle|\gamma|^{2}\right\rangle$ for the squiggles. In both cases there are no two-legged subdiagrams. However, although the equation $\left\langle\left|\gamma_{q}\right|^{2}\right\rangle=\frac{1}{1+\left\langle\pi_{q}\right\rangle}$ resums ladder or bubble diagrams (which is apparent from (4.57) or (4.26)) and more general four-legged particle subdiagrams if the terms for $r \geq 4$ in (4.72) are taken into account, the right hand side of $(4.71,72)$ still contains diagrams with fourlegged particle subdiagrams. Thus, the resummation of four-legged particle subdiagrams is only partially through the complete resummation of two-legged squiggle diagrams. Also observe that, in going from $(4.68,69)$ to $(4.70-72)$, we cut off the $r$-sum at some fixed order $\ell$ independent of the volume since we can only expect that the expansions are asymptotic ones, compare the discussion in section 2 . 


\section{Concluding Remarks}

In the general case, without making the approximation (4.65), we expect the following picture for a generic quartic field theoretical model. Let $G$ and $G_{0}$ be the interacting and free particle Greens function (one solid line goes in, one solid line goes out), and let $D$ and $D_{0}$ be the interacting and free interaction Greens function (one wavy line goes in, one wavy line goes out). Then we expect the following closed set of integral equations for $G$ and $D$ :

$$
G=\frac{1}{G_{0}^{-1}+\sigma(G, D)}, \quad D=\frac{1}{D_{0}^{-1}+\pi(G, D)}
$$

where $\sigma$ and $\pi$ are the sum of all two legged diagrams without two legged (particle and wavy line) subdiagrams with propagators $G$ and $D$ (instead of $G_{0}, D_{0}$ ). Thus (5.1) simply eliminates all two legged insertions by substituting them by the full propagators. For the Anderson model $D=D_{0}=1$ and (5.1) reduces to $(2.27,35)$.

A variant of equations (5.1) has been derived on a more heuristic level in [CJT] and [LW]. Their integral equation (for example equation (40) of [LW]) reads

$$
G=\frac{1}{G_{0}^{-1}+\tilde{\sigma}\left(G, D_{0}\right)}
$$

where $\tilde{\sigma}$ is the sum of all two legged diagrams without two legged particle insertions, with propagators $G$ and $D_{0}$. Thus this equation does not resum two legged interaction subgraphs (one wavy line goes in, one wavy line goes out). However resummation of these diagrams corresponds to a partial resummation of four legged particle subgraphs (for example the second equation in (5.4) below resums bubble diagrams), and is necessary in order to get the right behaviour, in particular for the many-electron system.

Another popular way of eliminating two legged subdiagrams (instead of using integral equations) is the use of counterterms. The underlying combinatorial identity is the following one. Let

$$
\mathcal{S}(\psi, \bar{\psi})=\int d k \bar{\psi}_{k} G_{0}^{-1}(k) \psi_{k}+\mathcal{S}_{\mathrm{int}}(\psi, \bar{\psi})
$$

be some action of a field theoretical model and let $T(k)=T\left(G_{0}\right)(k)$ be the sum of all amputated two legged particle diagrams without two legged particle subdiagrams, evaluated with the bare propagator $G_{0}$. Let $\delta \mathcal{S}(\psi, \bar{\psi})=\int d k \bar{\psi}_{k} T(k) \psi_{k}$. Consider the model with action $\mathcal{S}-\delta \mathcal{S}$. Then a $p$-point function of that model is given by the sum of all $p$-legged diagrams which do not contain any two legged particle subdiagrams, evaluated with the bare propagator $G_{0}$. In particular, by construction, the two point function of that model is exactly given by $G_{0}$. Now, since the quadratic part of the model under consideration (given by the action $\mathcal{S}-\delta \mathcal{S}$ ) should be given by the bare Greens function $G_{0}^{-1}$ and the interacting Greens function is $G$, one is led to the equation $G^{-1}-T(G)=G_{0}^{-1}$ which coincides with (5.2). 
Since the quantities $\sigma$ and $\pi$ in (5.1) are not explicitely given but merely are given by a sum of diagrams, we have to make an approximation in order to get a concrete set of integral equations which we can deal with. That is, we substitute $\sigma$ and $\pi$ by its lowest order contributions which leads to the system

$$
G(k)=\frac{1}{G_{0}(k)^{-1}+\int d p D(p) G(k-p)}, \quad D(q)=\frac{1}{D_{0}(q)^{-1}+\int d p G(p) G(p+q)}
$$

This corresponds to the use of $(1.3,4)$ retaining only the $r=2$ term. Thus we assume that the expansions for $\sigma$ and $\pi$ are asymptotic. A rigorous proof of that is of course a very difficult mathematical problem and this has not been adressed in this paper. Roughly one may expect this if each diagram contributing to $\sigma$ and $\pi$ allows a const ${ }^{n}$ bound (no $n$ ! and of course no divergent contributions). One may look in [FKLT] for an outline of proof for the many electron system with an anisotropic dispersion relation. In that case actually one obtains a series with a small positive radius of convergence instead of only an asymptotic one (because the model is fermionic), which simplifies the proof considerably.

Finally we remark that the equations (5.4) can be found in the literature. Usually they are derived from the Schwinger-Dyson equations which is the following non closed set of two equations for the three unknown functions $G, D$ and $\Gamma, \Gamma$ being the vertex function (see, for example, $[\mathrm{AGD}]$ ):

$$
\begin{aligned}
G(k) & =G_{0}(k)+G_{0}(k) \int d p G(p) D(k-p) \Gamma(p, k-p) G(k) \\
D(q) & =D_{0}(q)+D_{0}(q) \int d p G(p) G(p+q) \Gamma(p+q,-q) D(q)
\end{aligned}
$$

The function $\Gamma(p, q)$ corresponds to an off-diagonal inverse matrix element as it shows up for example in (4.22). Then application of (1.4) transforms (5.5) into (5.1). One may say that although the equations (5.4) are known, usually they are not really taken seriously. For our opinion this is due to two reasons. First of all these equations, being highly nonlinear, are not easy to solve. In particular, for models involving condensation phenomena like superconductivity or Bose-Einstein condensation, it seems to be apropriate to write them down in finite volume since some quantities may become macroscopic. And second, since they are usually derived from (5.5) by putting $\Gamma$ equal to 1 (or actually -1 , by the choice of signs in (5.5)), one may feel pretty suspicious about the validity of that approximation. The equations (5.1) tell us that this is a good approximation if the expansions for $\sigma$ and $\pi$ are asymptotic.

The applications of the method shown in this paper basically confirmed the common expectations for the particular models, thus one may say there are no really new results. However, we think it is fair to say that the computation of field theoretical correlation functions is an extremely difficult mathematical problem and therefore one should have 
welcome everything which sheds some new light on these problems. We hope that we could convince the reader that the method presented in this paper definitely does this.

\section{References}

[AG] M. Aizenman, G.M. Graf, Localization Bounds for an Electron Gas, Journ. Phys. A, Bd. 31, S. 6783, 1998.

[AGD] A. A. Abrikosov, L. P. Gorkov, I. E. Dzyaloshinski, Methods of Quantum Field Theory in Statistical Physics, Prentice-Hall, 1963.

[B] N. N. Bogoliubov, Lectures on Quantum Statistics, Vol.2, Gordon and Breach, 1970

[BFS] V. Bach, J. Fröhlich, and I. M. Sigal, Renormalization group analysis of spectral problems in quantum field theory, Adv. in Math. 137, 205-298, 1998.

[CFS] T. Chen, J. Fröhlich, M. Seifert, Renormalization Group Methods: Landau-Fermi Liquid and BCS Superconductor, Proceedings of the Les Houches session Fluctuating Geometries in Statistical Mechanics and Field Theory, eds. F. David, P. Ginsparg, J. Zinn-Justin, 1994.

[CJT] J. M. Cornwall, R. Jackiw, E. Tromboulis, Effective Action for Composite Operators, Phys. Rev. D, vol.10, no.8, p.2428-2445, 1974.

[F] J. Fröhlich, On the Triviality of $\lambda \varphi_{d}^{4}$ Theories and the Approach to the Critical Point, Nucl. Physics B200, 281-296, 1982.

[FFS] R. Fernandez, J. Fröhlich, A. Sokal, Random Walks, Critical Phenomena and Triviality in Quantum Field Theory, Texts and Mongraphs in Physics, Springer 1992.

[FKLT] J. Feldman, H. Knörrer, D. Lehmann, E. Trubowitz, Fermi Liquids in Two Space Dimensions, in Constructive Physics, Springer Lecture Notes in Physics, Bd. 446, 1994.

[FKT] J. Feldman, H. Knörrer, E. Trubowitz, Mathematical Methods of Many Body Quantum Field Theory, Lecture Notes, ETH Zürich.

[FMRT] J. Feldman, J. Magnen, V. Rivasseau, E. Trubowitz, Ward Identities and a Perturbative Analysis of a U(1) Goldstone Boson in a Many Fermion System, Helvetia Physica Acta 66, 1993, 498-550. 
[FT] J. Feldman, E. Trubowitz, Perturbation Theory for Many Fermion Systems, Helvetia Physica Acta 63, p.156-260, 1990; The Flow of an Electron-Phonon System to the Superconducting State, Helv. Phys. Acta 64, p.214-357, 1991.

[K] A. Klein, The Supersymmetric Replica Trick and Smoothness of the Density of States for Random Schrödinger Operators, Proceedings of Symposia in Pure Mathematics, vol. 51, part 1, 1990.

[L1] D. Lehmann, The Many-Electron System in the Forward, Exchange and BCS Approximation, Comm. Math. Phys. 198, 427-468, 1998.

[L2] D. Lehmann, The Global Minimum of the Effective Potential of the Many-Electron System with Delta-Interaction, to appear in Rev. Math. Phys. Vol. 12, No 9, Sept. 2000 .

[LW] J. M. Luttinger, J. C. Ward, Ground State Energy of a Many-Fermion System II, Phys. Rev. vol. 118, no. 5, p.1417-1427, 1960.

[MPR] J. Magnen, G. Poirot, V. Rivasseau, Ward Type Identities for the 2D Anderson Model at Weak Disorder, cond-mat/9801217; The Anderson Model as a Matrix Model, Nucl. Phys. B (Proc. Suppl.) 58, S.149, 1997.

[P] G. Poirot, Mean Green's Function of the Anderson Model at Weak Disorder with an Infrared Cutoff, cond-mat/9702111

[R] V. Rivasseau, From Perturbative to Constructive Renormalization, Princeton Univ. Press 1991.

[W] Wei-Min Wang, Supersymmetry, Witten Complex and Asymptotics for Directional Lyapunov Exponents in $\mathbb{Z}^{d}$, mp-arc/99-355; Localization and Universality of Poisson Statistics Near Anderson Transition, mp-arc/99-473. 